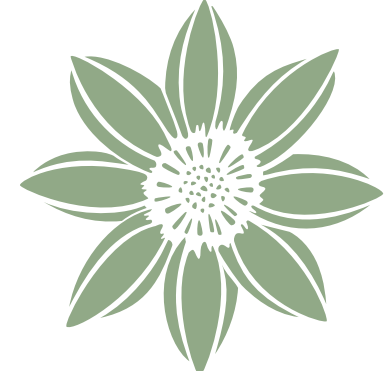

Acta Botanica Mexicana

\title{
Variación en la estructura del bosque de Abies religiosa (Pinaceae), en diferentes condiciones de manejo y disturbio
}

\section{Variation in the Abies religiosa (Pinaceae) forest structure,} at different management and disturbance conditions

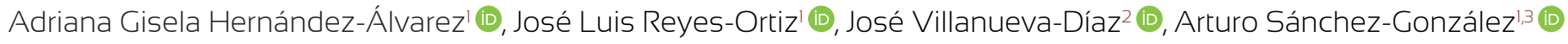

\section{Resumen:}

Antecedentes y Objetivos: El bosque de Abies religiosa o bosque de oyamel se distribuye en parches aislados y sus áreas continuas más extensas se localizan en la Faja Volcánica Transmexicana. Diversos estudios aportan información sobre aspectos ecológicos y florísticos del bosque de oyamel en México. Sin embargo, aún es necesario responder cuestiones ecológicas básicas, que pueden ser útiles para proponer y establecer estrategias de manejo y conservación de este tipo de vegetación. Por esta razón, los objetivos de la presente investigación fueron: (1) estimar la variación en la estructura poblacional de Abies religiosa y (2) determinar la estructura y composición arbórea y arbustiva del bosque de oyamel bajo diferentes condiciones de manejo.

Métodos: El área de estudio incluyó cinco localidades con bosque de oyamel del estado de Hidalgo, México, con diferentes condiciones de manejo y altitud. En total se colocaron 25 parcelas de muestreo ( 0.01 ha cada una) para obtener datos estándar a nivel poblacional (altura, densidad, diámetro) y comunitario (densidad, área basal y frecuencia de las especies).

Resultados clave: Dentro de las cinco localidades, tres poblaciones de A. religiosa se clasificaron como dinámicas, por presentar predominio de individuos pequeños; las dos restantes fueron normales, con alto porcentaje de plantas de tamaño intermedio. A nivel de comunidad, se registraron 15 especies de árboles y 15 de arbustos. La estructura del bosque difirió entre localidades, en especial las más lejanas geográficamente, corroborando el papel del manejo del bosque y de las condiciones ambientales locales.

Conclusiones: Abies religiosa dominó el dosel en todas las localidades. Las características estructurales a nivel de población y comunidad difirieron entre sitios de muestreo probablemente a causa del manejo forestal y de las diferentes condiciones ambientales locales.

Palabras clave: conservación, dominancia, Faja Volcánica Transmexicana, oyamel, población dinámica, riqueza de especies.

\section{Abstract:}

Background and Aims: Abies religiosa forest or sacred fir forest is distributed in isolated patches and its largest continuous areas are located in the Transmexican Volcanic Belt. There are several studies that provide essential information on ecological and floristic aspects of the sacred fir forest in Mexico. However, basic ecological questions still need to be answered, which can be very useful to propose and establish management and conservation strategies for this type of vegetation. For this reason, the objectives of this investigation were: (1) to estimate the variation in the population structure of Abies religiosa and, (2) determine the tree and shrub structure and composition of the sacred fir forest, under different conditions of management and elevation.

Methods: The study area included five localities with sacred fir forest in Hidalgo State, with different management and altitude conditions. In total, 25 sampling plots were placed ( 0.01 ha each), to obtain standard data at the population level (height, density, diameter) and community level (density, basal area and frequency of species).

Key results: Three populations of $A$. religiosa were classified as dynamic due to the predominance of small individuals. The remaining two were normal with a predominance of intermediate-sized specimens. At the community level, 15 species of trees and 15 of shrubs were recorded. The structure of the forest differed in some localities, mainly among the farthest geographically, confirming the role of forest management, and local environmental conditions.

Conclusions: Abies religiosa dominated the canopy in all localities. The structural characteristics at both population and community levels differed between sampling locations, probably due to the forest management, and the different local environmental conditions.

Key words: conservation, dominance, dynamic population, sacred fir, species richness, Transmexican Volcanic Belt.

${ }^{1}$ Universidad Autónoma del Estado de Hidalgo, Centro
de Investigaciones Biológicas, Ciudad del Conocimien-
to, Carretera Pachuca-Tulancingo km 4.5, 42184 Mi-
neral de la Reforma, Hidalgo, México.
${ }^{2}$ Instituto Nacional de Investigaciones Forestales Agrí-
colas y Pecuarias, Centro Nacional de Investigación
Disciplinaria en Relación Agua, Suelo, Planta, Atmós-
fera, km 6.5 Margen Derecha del Canal Sacramento,
35140 Gómez Palacio, Durango, México.
${ }^{3}$ Autor para la correspondencia: arturosg@uaeh.edu.mx
Recibido: 12 de julio de 2020.

Revisado: 11 de agosto de 2020.

Aceptado por Pablo Cuevas-Reyes: 3 de febrero de 2021.

Publicado Primero en línea: 11 de febrero de 2021.

Publicado: Acta Botanica Mexicana 128(2021).

(7) (8) Este es un artículo de acceso abierto bajo la licencia Creative Commons 4.0 Atribución-No Comercial (CC BY-NC 4.0 Internacional).
Citar como: Hernández-Álvarez, A. G., J. L. ReyesOrtiz, J. Villanueva-Díaz y A. Sánchez-González. 2021. Variación en la estructura del bosque de Abies religiosa (Pinaceae), en diferentes condiciones de manejo y disturbio. Acta Botanica Mexicana 128: e1752. DOI: https://doi.org/10.21829/abm128.2021.1752 


\section{Introducción}

El bosque de Abies religiosa (Kunth) Cham. \& Schltdl., o bosque de oyamel, ocupa entre 0.07 y $0.16 \%$ de la superficie de México y su distribución se presenta en forma de parches aislados (Flores-Mata et al., 1971; Rzedowski, 1998; INEGI, 2017). Es un bosque típico de la zona ecológica templada subhúmeda (sensu Toledo et al., 1993), con características florísticas y ecológicas particulares asociadas a factores climáticos y edáficos; se desarrolla en zonas montañosas en altitudes entre 2400 y 3500 m (Rzedowski, 1978; Sánchez-González y López-Mata, 2005; Farjon, 2010). Las áreas continuas más extensas de este tipo de vegetación se localizan en la Faja Volcánica Transmexicana (FVT, sensu Ferrusquía-Villafranca, 1998) donde A. religiosa domina el estrato arbóreo superior (SánchezGonzález et al., 2005).

Diferentes estudios ecológicos y florísticos han contribuido con información primordial para una mejor comprensión del bosque de oyamel en México. Desde el punto de vista ecológico el bosque de Abies L. brinda servicios ecosistémicos como la protección contra la erosión, captura de carbono, captación de agua y regulación de la escorrentía (Hernández, 1985; Kasischke y Stocks, 2000). Además, provee beneficios económicos directos, por ejemplo, la madera de oyamel contiene altos niveles de celulosa y es empleada en la fabricación de papel fino que alcanza elevados precios en el mercado (Rodríguez-Laguna et al., 2015). Otros aportes se han centrado en la caracterización de las condiciones ecológicas que favorecen la distribución y el desarrollo de este tipo de vegetación (Madrigal, 1967; Sánchez-Velásquez et al., 1991), el reclutamiento y la estructura poblacional en la región del Cofre de Perote, Veracruz (Lara-González et al., 2009; Pineda-López et al., 2013) y en Tlaxco, Tlaxcala (Bautista, 2013), el análisis cualitativo de su hábitat en Hidalgo (García, 2002), la supervivencia de las plántulas en el Parque Nacional El Chico, Hidalgo (Ángeles-Cervantes y López-Mata, 2009), la migración de los individuos de oyamel hacia altitudes más elevadas (Flores Nieves, 2015), la migración asistida en el Estado de México (Carbajal-Navarro et al., 2019), la predicción de su distribución en diferentes escenarios de cambio climático (Sáenz-Romero et al., 2012; Pérez Miranda et al., 2014) y la influencia de la temperatura y precipitación sobre el creci- miento radial (Carlón-Allende et al., 2016; Aquino-Ramírez et al., 2019).

Florísticamente, la descripción de las principales especies vegetales del bosque de oyamel que se distribuye en la FVT (Rzedowski y Calderón de Rzedowski, 1979; Hernández, 1995), ha permitido analizar la semejanza entre distintos fragmentos distribuidos a lo largo de esta región biogeográfica (Sánchez-González et al., 2005). En otros estudios florísticos se analizó la distribución de especies de los bosques de oyamel de la Sierra Nevada (Sánchez-González et al., 2006) y del Parque Estatal Santuario de Agua Presa Corral de Piedra, ambos en el Estado de México (ZepedaGómez et al., 2018). Con base en las contribuciones florísticas previas se estima que la riqueza del bosque de oyamel se integra por 510 especies distribuidas en 222 géneros y 76 familias (Sánchez-González et al., 2005). Sin embargo, su composición de especies arbóreas, arbustivas y herbáceas puede variar entre fragmentos de bosque, dependiendo de los cambios en la topografía, altitud, intervención humana u otras causas (Challenger, 1998; Sánchez-González y López Mata, 2003; Farjon, 2010).

En este sentido, el bosque maduro de oyamel, al igual que la mayoría de los bosques de coníferas de México, ha sufrido una drástica disminución en su área de distribución y cambios en composición y estructura ecológica (SánchezVelásquez et al., 1991; Ángeles-Cervantes y López-Mata, 2009; Pineda-López et al., 2013; Razo-Zárate et al., 2013). Entre los factores que provocan la transformación de los bosques primarios de oyamel destacan la alta frecuencia de incendios (naturales y provocados por el hombre), tala inmoderada (aprovechamiento directo de la madera y productos secundarios y la expansión de la actividad agrícola y pecuaria), contaminación del aire, ausencia de manejo forestal, plagas, patógenos y el cambio climático (Alvarado et al., 1993; Sáenz-Romero et al., 2012; Pérez Miranda et al., 2014; González Hernández et al., 2015; Carlón-Allende et al., 2016). Actualmente, a pesar de la importancia ecológica y de las diversas fuentes de perturbación que afectan al bosque de oyamel, la especie dominante Abies religiosa, se mantiene en la categoría de "preocupación menor" (LC) en la Lista Roja de la IUCN (IUCN, 2020).

Por lo tanto, es preciso generar información básica sobre el efecto de los cambios ambientales en la compo- 
sición y estructura del bosque de oyamel, para definir o modificar las estrategias de uso y conservación del mismo y, por ende, de la especie Abies religiosa. Por lo anterior, los objetivos de la presente investigación fueron: a) estimar el cambio en la estructura poblacional de Abies religiosa y b) determinar la estructura y composición arbórea y arbustiva del bosque de oyamel bajo diferentes condiciones de manejo, tomando como modelo de estudio el bosque del estado de Hidalgo, México.

\section{Materiales y Métodos}

\section{Área de estudio}

El bosque de oyamel del estado de Hidalgo se localiza al interior de la Faja Volcánica Transmexicana, entre las coordenadas extremas $19^{\circ} 56^{\prime}-20^{\circ} 13^{\prime} \mathrm{N}$ y $98^{\circ} 21^{\prime}-98^{\circ} 48^{\prime} \mathrm{O}$, en un intervalo altitudinal entre 2600 y 3200 m s. n. m. (BarriosRodríguez y Medina-Cota, 1996; CONANP, 2006). En general, el clima predominante es $\mathrm{Cb}(\mathrm{m})(\mathrm{w})(\mathrm{i}) \mathrm{gw}$ ": templado sub-húmedo con verano fresco y largo (García, 2004), temperatura media anual entre 12 y $18{ }^{\circ} \mathrm{C}$ y precipitación media anual superior a 1000 mm (Pavón y Meza, 2009).

Las localidades de muestreo situadas en el estado de Hidalgo fueron el Parque Nacional El Chico (PNEC, municipios Mineral del Chico y Pachuca), ejido El Cerezo (Pachuca), ejido Pueblo Nuevo (Mineral del Monte), ejido Xolostitla (Epazoyucan) y ejido Tecocomulco (Cuautepec de Hinojosa y Singuilucan) (Cuadro 1, Fig. 1). Las características ambientales en las localidades de muestreo fueron heterogéneas, debido a que presentan distintas condiciones de manejo, altitud y extensión territorial (Galindo-Leal et al., 1988; Sánchez-González et al., 2005; CONANP, 2006; Rodríguez-Laguna et al., 2015).

\section{Análisis estructural}

En el análisis de la estructura poblacional de Abies religiosa y de la estructura del bosque de oyamel se seleccionaron al azar cinco unidades de muestreo (UM) de $20 \times$ 50 m cada una por localidad; la distancia mínima entre UM fue de $60 \mathrm{~m}$. El tamaño de área de la UM utilizada (0.01 ha) ha permitido obtener una representación apropiada de la composición y estructura de los bosques en estudios previos (Gentry, 1982; Trejo y Dirzo, 2002; Sánchez-González y López-Mata, 2003; Bravo-Bolaños et al., 2016; Ortiz-Quijano et al., 2016).

\section{Estructura poblacional de Abies religiosa}

Las poblaciones se caracterizaron en función del diámetro normal a $1.30 \mathrm{~m}$ de altura (DN) de todos los individuos con diámetro $\geq 1 \mathrm{~cm}$ (medido con cinta diamétrica) y de la altura total de los mismos (medida con un hipsómetro Nikon Forestry Pro, Tokio, Japón), en cada UM y por localidad de estudio. El número de clases de altura y de diámetro se determinó utilizando la regla de Sturges: $k=1+3.322$ $\left(\log _{10} n\right)$, donde k=número de grupos (intervalos de clase) y $\mathrm{n}=$ número de individuos (Schmidt et al., 2009). Con el propósito de realizar un análisis comparativo de la estructura poblacional entre localidades, se eligió el mismo número y ancho de clase de los intervalos de altura y diámetro para las cinco localidades, tomando como referencia para hacer el cálculo a la localidad con mayor número de individuos (Pérez-Paredes et al., 2014). La amplitud de los intervalos de clase (Al) se estimó con la fórmula $A \mathrm{I}=R / k$, donde $R=$ rango y $k$ es el número de intervalos de clase (Daniel, 1995). La estimación de la relación entre altura y diámetro de los individuos se realizó mediante análisis de regresión

Cuadro 1: Características generales de las cinco localidades de muestreo con bosque de Abies religiosa (Kunth) Cham. et Schltdl. de Hidalgo, México. Condición de manejo: MFI: manejo forestal intensivo, MFM: manejo forestal moderado, MFL: manejo forestal leve. * La información sobre la condición de manejo se incluye en el Cuadro 2.

\begin{tabular}{ccccccc}
\hline & Localidad & Extensión (ha) & Latitud (N) & Longitud (O) & Altitud (m) & Condición* \\
\hline A & Pueblo Nuevo & 1783.14 & $20^{\circ} 10^{\prime} 53.8^{\prime \prime}$ & $98^{\circ} 40^{\prime} 00.8^{\prime \prime}$ & 2975 & $\mathrm{MFI}$ \\
B & PNEC & 1725.4 & $20^{\circ} 11^{\prime} 20.5^{\prime \prime}$ & $98^{\circ} 42^{\prime} 57.7^{\prime \prime}$ & 2974 & $\mathrm{MFL}$ \\
C & Tecocomulco & 381.09 & $19^{\circ} 56^{\prime} 53.1^{\prime \prime}$ & $98^{\circ} 21^{\prime} 41.7^{\prime \prime}$ & 2904 & $\mathrm{MFM}$ \\
D & El Cerezo & 360 & $20^{\circ} 10^{\prime} 57.9^{\prime \prime}$ & $98^{\circ} 42^{\prime} 39.3^{\prime \prime}$ & 2949 & $\mathrm{MFL}$ \\
E & Xolostitla & 162.78 & $20^{\circ} 06^{\prime} 49.2^{\prime \prime}$ & $98^{\circ} 37^{\prime} 26.8^{\prime \prime}$ & 2861 & $\mathrm{MFM}$ \\
\hline
\end{tabular}



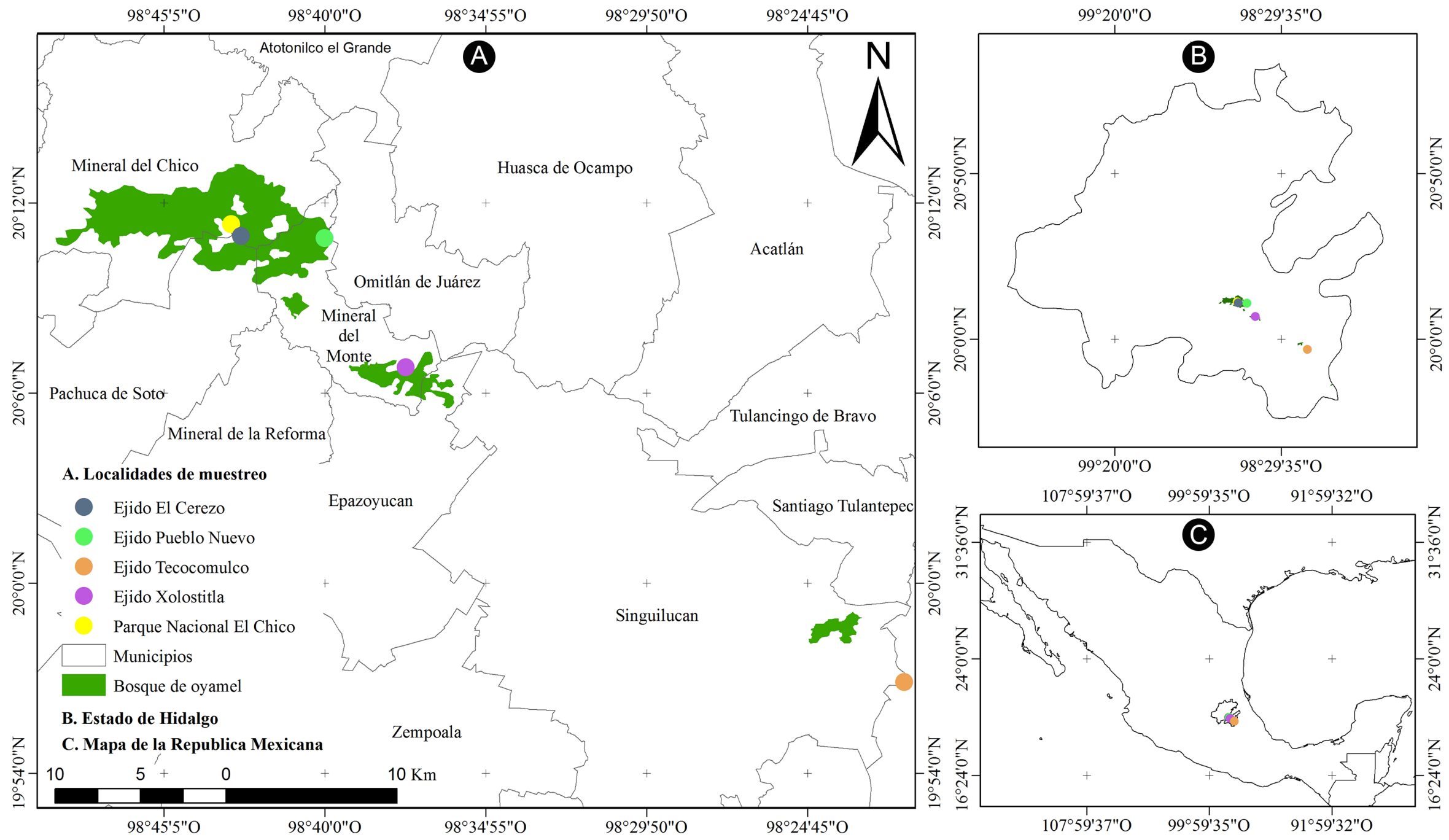

Figura 1: Mapa de distribución de las localidades con bosque de Abies religiosa (Kunth) Schltdl. \& Cham., analizadas en el estado de Hidalgo, México. A. localidades de muestreo; B. estado de Hidalgo; C. mapa de la República Mexicana. 
lineal y no lineal; de los modelos resultantes, se eligió el que resultó con valor de coeficiente de determinación $\left(r^{2}\right)$ más alto (Urban et al., 2010). Los cálculos se llevaron a cabo utilizando el programa STATISTICA v. 13.0 (StatSoft, 2015).

Las poblaciones de oyamel se clasificaron en dinámicas, normales (estándar) o regresivas, de acuerdo con la altura de los individuos (Aguraiuja et al., 2004; Pérez-Paredes et al., 2014). Esta clasificación provee una evaluación demográfica adecuada del estado actual de una especie (Hegland et al., 2001). Si la estructura de tamaños de una población presentó un alto número de individuos pequeños (plantas <13 m de altura) se clasificó como dinámica; aquellas poblaciones con individuos de alturas intermedias (13-36 m de altura) se consideraron como normales o estándar, mientras que las poblaciones regresivas se caracterizaron por el predominio de árboles altos (>36 m) y escasos individuos de talla intermedia o pequeña. Por otra parte, para definir si existían diferencias en la regeneración del bosque entre las localidades, se realizó el conteo del número de plántulas de oyamel en cada UM; se consideró arbitrariamente como plántula a todos los individuos con altura $<10 \mathrm{~cm}$.

\section{Estructura del bosque de oyamel}

En cada una de las UM se recolectaron ejemplares de árboles y arbustos y posteriormente se determinaron a nivel de especie con claves dicotómicas específicas para cada género (Calderón de Rzedowski y Rzedowski, 2001; Valencia-A. et al., 2017). La nomenclatura de las especies fue actualizada de acuerdo con la base de datos del IPNI (2020) y TROPICOS (2020).

Únicamente los individuos con altura $>1.5 \mathrm{~m}$ y diámetro a la altura del pecho (DAP a $1.3 \mathrm{~m}$ de altura) $>2.5$ cm (Ortiz-Quijano et al., 2016) fueron registrados y considerados para el análisis. Con los datos obtenidos se estimó la densidad (D), definida como el número de individuos de cada especie por unidad de superficie de muestreo. La densidad relativa (DR) se calculó como la densidad de individuos por especie entre el número total de individuos de todas las especies. La frecuencia se estimó como el número de UM con presencia de individuos de una especie entre el total de UM por localidad. La dominancia se obtuvo como la función del área basal $\left(\mathrm{m}^{2} \mathrm{ha}^{-1}\right)$ de cada especie de árbol y arbusto (Lamprecht, 1986; Rozas, 2002; Sánchez-Rodríguez et al., 2003; Bianchi et al., 2011), a partir de la fórmula $\pi r^{2}$, donde $r$ es el radio, que se calcula como $r=1 / 2$ del diámetro del árbol o arbusto. El área basal relativa (ABR) se determinó como el área basal de cada especie entre área basal total en cada UM y por localidad (Lamprecht, 1986).

La estructura del bosque se describió a partir del valor de importancia relativa (VIR) de cada especie (Curtis y McIntosh, 1951). EI VIR se estimó sumando la DR, FR y ABR y dividiendo el valor de la suma entre tres (Mueller-Dombois y Ellenberg, 1974; Matteucci y Colma, 1982).

\section{Similitud florística entre localidades}

La similitud entre localidades fue estimada por medio de análisis de agrupamiento empleando los datos de VIR de las especies. El análisis se efectuó en el programa PAST v. 3.03 (Hammer et al., 2001), donde se eligió el índice de BrayCurtis como la medida de semejanza y el promedio entre grupos o UPGMA como el método de unión (Matteucci y Colma, 1982; Sánchez-González y López-Mata, 2003). EI dendrograma resultante permitió establecer el número mínimo de grupos homogéneos, a partir de un nivel de corte arbitrario que describe la relación topológica entre las localidades con bosque de oyamel, desde un punto de vista ecológico (McCune y Grace, 2002). Una vez obtenidos los grupos que permitieron describir la variación en la composición de especies y en la estructura del bosque de oyamel, cada uno se representó por medio de un diagrama de perfil semi-realista (Sánchez-González y López-Mata, 2003; Bravo Bolaños et al., 2016).

\section{Grado o nivel de manejo y disturbio}

La estimación del manejo forestal y el disturbio fue cualitativa, se basó en los siguientes parámetros: 1) información general de la condición de manejo, proporcionada por los guías en cada localidad, 2) observación en campo de presencia de caminos, árboles talados, aclareo, entre otras características y 3) se consideró a priori que existe asociación negativa entre el nivel de manejo y la densidad poblacional de Abies religiosa (Santibañez-Andrade et al., 2015).

Con la información anterior, las localidades se clasificaron en tres categorías, de acuerdo con las evidencias detectadas durante el trabajo en campo: a) con manejo fo- 
restal leve o sin manejo: localidad dentro de un área natural protegida (ANP), en la zona núcleo, con restricciones de acceso al público; b) con manejo forestal moderado: localidades sin restricción de acceso a personas (ecoturismo), y c) con manejo forestal intensivo: control total de acceso al bosque por los ejidatarios (Cuadro 2).

\section{Resultados}

\section{Estructura poblacional por clases de altura y diámetro}

La densidad de la población de $A$. religiosa ubicada en el PNEC fue de 454 individuos ha ${ }^{-1}$ y los promedios de altura y diámetro fueron de $16.55 \pm 13.97 \mathrm{~m}$ y $27.74 \pm 26.83 \mathrm{~cm}$, respectivamente. En "El Cerezo" la densidad fue de 452 individuos ha-1 ${ }^{-1}$ con altura y diámetro promedio de $23.37 \pm 13.72$ m y $32.44 \pm 23.48 \mathrm{~cm}$, respectivamente. El valor de densidad más bajo se encontró en Pueblo Nuevo, con 258 individuos ha $^{-1}$; el promedio de altura y diámetro fue de $28.59 \pm 10.34$ m y $43.87 \pm 20.93 \mathrm{~cm}$, respectivamente. En Xolostitla se registraron 600 individuos $\mathrm{ha}^{-1}$, la altura y diámetro promedio fueron de $11.72 \pm 10.48 \mathrm{~m}$ y $16.89 \pm 23.14 \mathrm{~cm}$, respectivamente. La densidad más alta se registró en Tecocomulco con 950 árboles ha-1 y la altura y diámetro promedio de los árboles fue de $15.08 \pm 9.33 \mathrm{~m}$ y $16.71 \pm 18.97 \mathrm{~cm}$, respectivamente (Fig. 2).

La estructura de tamaños (altura) y diámetros en las poblaciones analizadas fue variable. Las poblaciones de Abies religiosa del PNEC, ejido Xolostitla y Tecocomulco presentaron una estructura en forma de $J$ invertida, en la que predominaron individuos de las primeras clases de altura y diámetro (Fig. 2), por lo que pueden considerarse como poblaciones dinámicas o en desarrollo. En cambio, la estructura de tamaños y diámetros fue diferente en las poblaciones de El Cerezo y Pueblo Nuevo, donde predominaron individuos de clases intermedias, por lo que se clasificaron como poblaciones normales (Fig. 2).

La relación entre la altura y diámetro de los individuos de las poblaciones analizadas fue altamente significativa $(P<0.001)$ para cada una de las localidades, con valores de r que oscilaron entre 0.67 y 0.92 . Cuando los datos de las localidades se analizaron en conjunto el valor de $r$ también fue altamente significativo (Fig. 3).

Por otra parte, en el total de las UM se contabilizaron 1984 individuos (plántulas) con altura $<10 \mathrm{~cm}$, distribuidos de la siguiente manera: $45.92 \%$ (911) en Pueblo Nuevo, $36.59 \%$ (726) en Xolostitla, 7.61\% (151) en El Cerezo, 5.04\% (100) en Tecocomulco y $4.84 \%$ (96) en el PNEC.

\section{Estructura y composición del bosque de oyamel} Se encontraron 30 especies (15 de árboles y 15 de arbustos), con importancia estructural en el bosque de oyamel analizado, que pertenecen a 24 géneros y 19 familias (Apéndice). La especie dominante fue Abies religiosa con VIR $>50 \%$ en todas las localidades, en Xolostitla fue don-

Cuadro 2: Criterios cualitativos para la clasificación del bosque de Abies religiosa (Kunth) Cham. \& Schltdl. en cada localidad muestreada en el estado de Hidalgo, México, de acuerdo con el grado de manejo y disturbio.

\begin{tabular}{lll}
\hline Manejo forestal & Criterios cualitativos de manejo y disturbio & Acceso \\
\hline Leve o sin manejo & $\begin{array}{l}\text { Zona núcleo de ANP, con vigilancia continua y escasas } \\
\text { labores de aclareo, claros nulos o escasos (por caída de }\end{array}$ & Restringido: acceso a turistas solo en caminos ex profeso \\
& árboles viejos), densidad poblacional de Abies L. alta. & \\
Moderado & Pastoreo, tala, aclareo, caminos de terracería antiguos & \\
& (tierra compactada), claros grandes escasos. Densidad & Libre: los ejidatarios permiten el acceso de turistas \\
& poblacional de Abies L. intermedia. & \\
Intensivo & Pastoreo, tala, aclareo, caminos de terracería antiguos & \\
& y recientes, presencia constante de personas y huellas & \\
& de vehículos cerca o dentro del bosque, claros grandes y & Restringido: ejidatarios controlan acceso \\
& frecuentes, poblaciones de especies de plantas indicadoras & \\
de disturbio abundantes. Densidad poblacional de Abies L. & \\
\hline
\end{tabular}




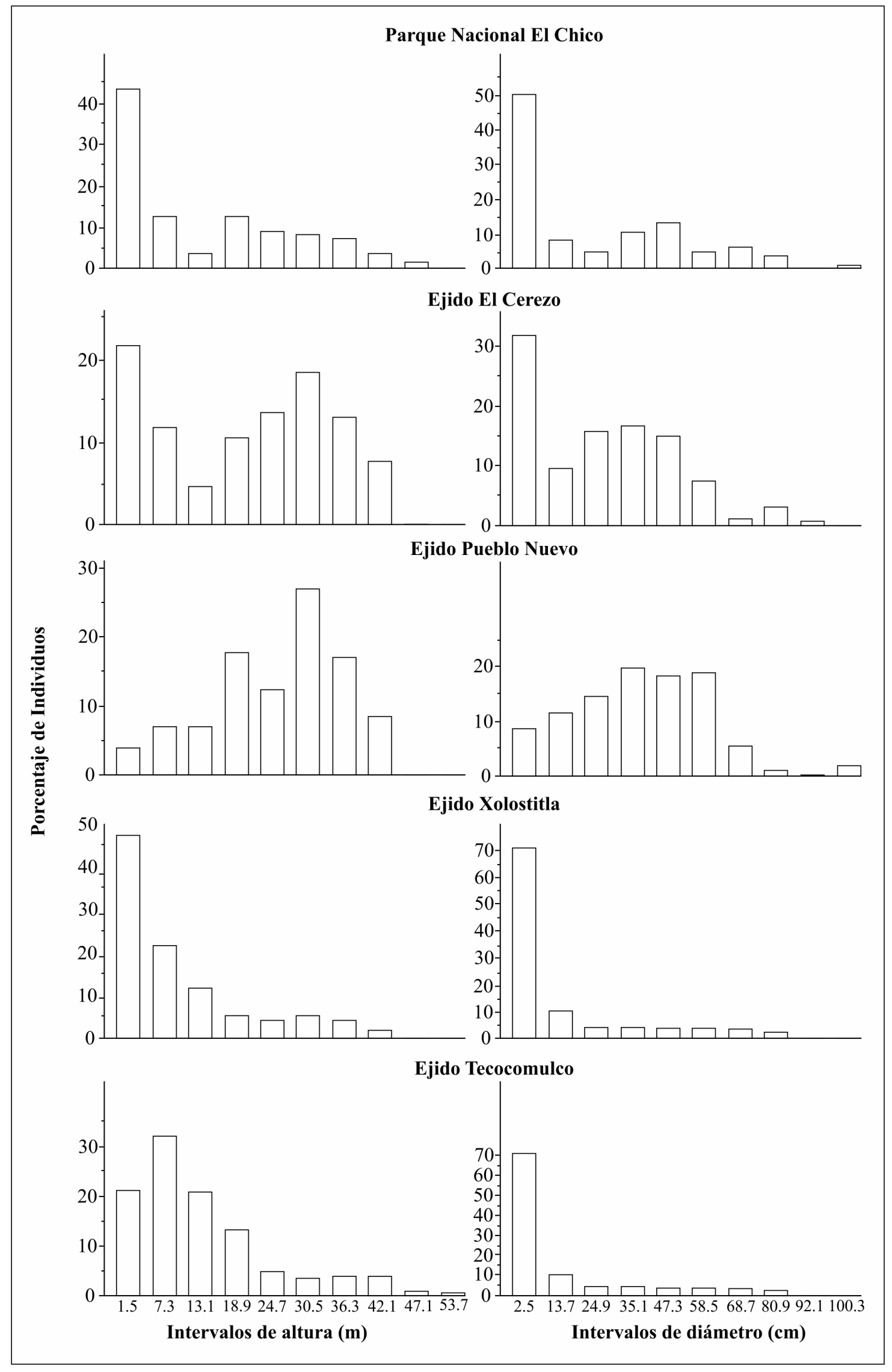

Figura 2: Clases de altura y diámetro de las cinco poblaciones de Abies religiosa (Kunth) Schltdl. \& Cham., analizadas en el estado de Hidalgo, México. 


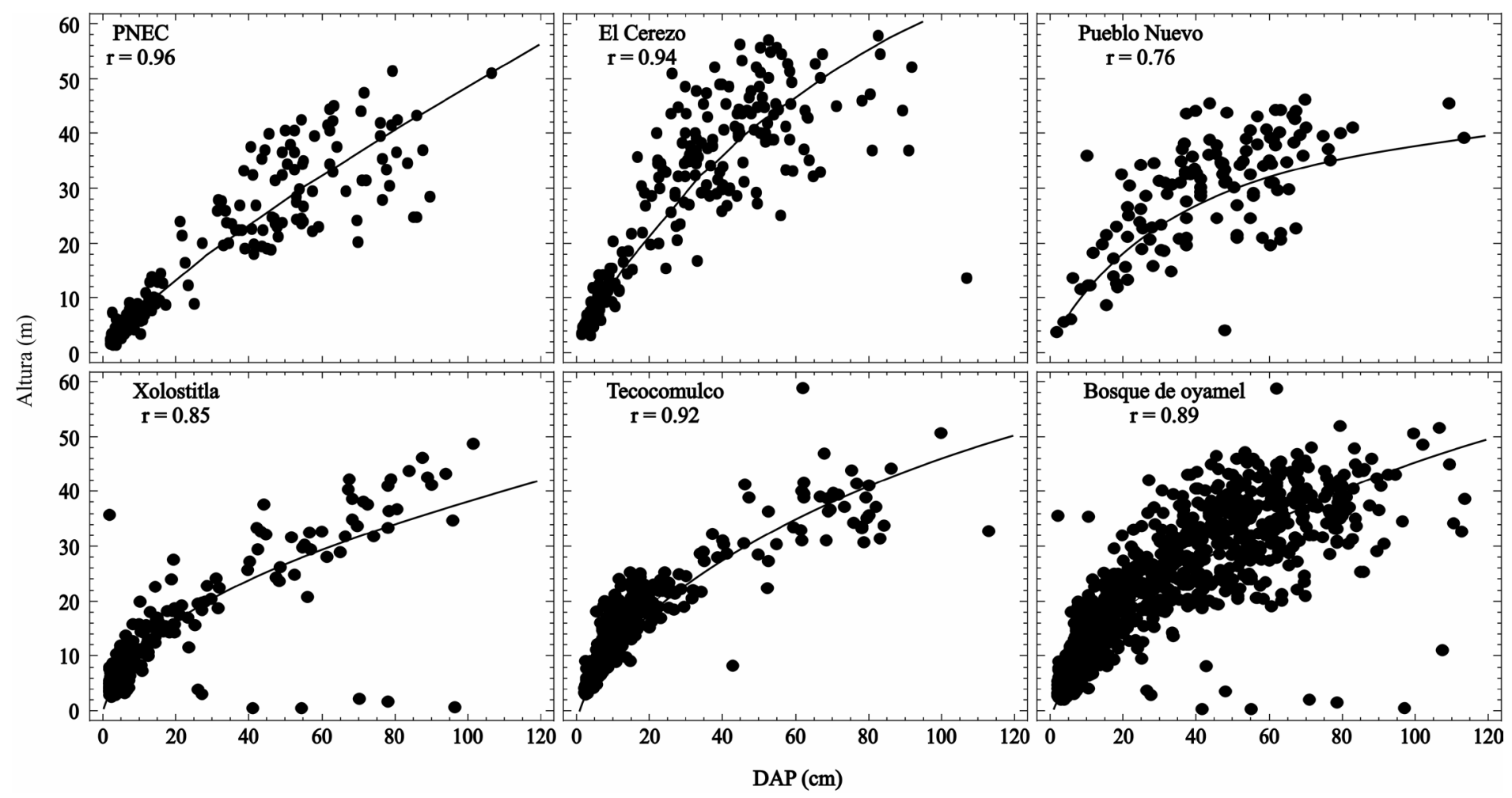

Figura 3: Correlación entre altura y diámetro de los individuos de distintas poblaciones de Abies religiosa (Kunth) Schltdl. \& Cham. Parque Nacional El Chico=PNEC, Diámetro a la altura del pecho=DAP. En todos los casos $\mathrm{P}<0.001$.

de se registró el valor más alto con 68.39\%. Las especies Juniperus monticola Martínez, Quercus glabrescens Benth., Pinus teocote Schltdl. \& Cham. y Quercus laurina Bonpl. fueron codominantes, con valores de VIR entre 6 y $11 \%$. Alnus jorulensis Kunth, algunas de las especies de Quercus L. y Archibaccharis serratifolia (Kunth) S.F. Blake se registraron solamente en una localidad (Cuadro 3). La riqueza de especies varió entre siete y 18 por localidad; Xolostitla presentó la menor riqueza y Pueblo Nuevo el valor más alto.
El análisis de agrupamiento indicó que la composición florística entre localidades fue semejante, pues el índice de Bray-Curtis osciló entre 0.62 y 0.75 . Se reconocieron cuatro grupos. El primero formado por la zona núcleo del PNEC y El Cerezo, el segundo por Pueblo Nuevo, el tercero por Xolostitla y el último por Tecocomulco (Fig. 4). A continuación, se describe con detalle la composición y estructura de cada uno de los grupos identificados:

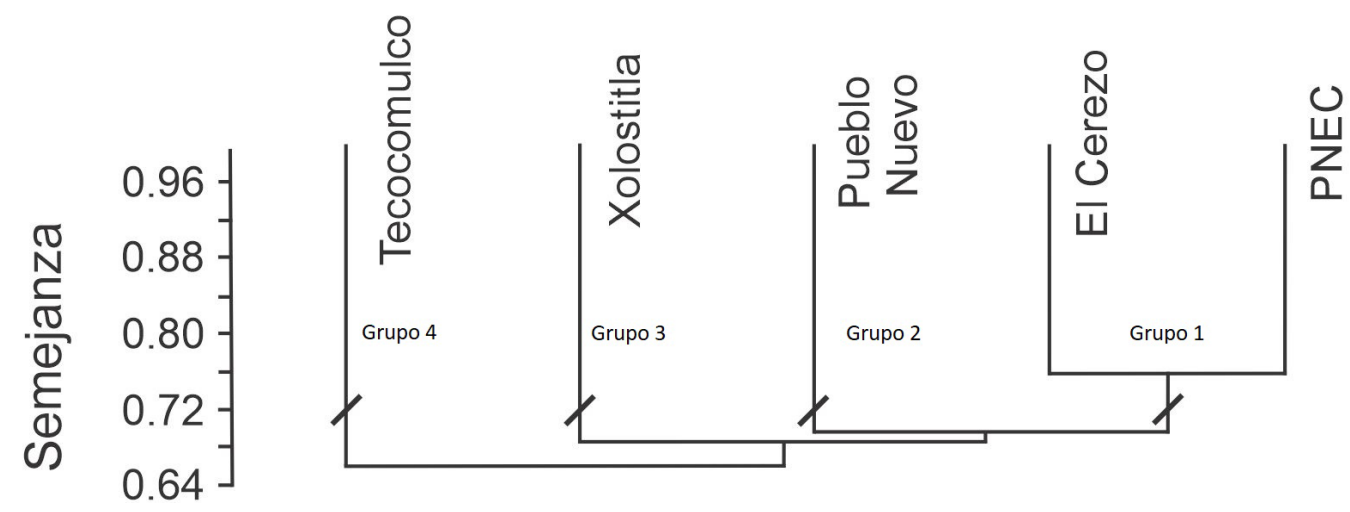

Figura 4: Dendrograma que muestra la semejanza en la composición de especies y la estructura del bosque de Abies religiosa (Kunth) Schltdl. \& Cham. de los sitios de muestreo en el estado de Hidalgo, México. 
Cuadro 3: Especies de árboles y arbustos del bosque de Abies religiosa (Kunth) Cham. \& Schltdl. con VIR más altos ( $>3 \%$ ) en las cinco localidades de muestreo en el estado de Hidalgo, México. D=Densidad; DR=Densidad relativa; AB=Área basal; ABR=Área basal relativa; FR. Frecuencia relativa; VIR=Valor de importancia relativa.

\begin{tabular}{|c|c|c|c|c|c|c|}
\hline & $\begin{array}{c}\text { D } \\
\text { (ind/ha) }\end{array}$ & $\begin{array}{l}\text { DR } \\
\text { (\%) }\end{array}$ & $\begin{array}{c}A B \\
\text { (m/ha) }\end{array}$ & $\begin{array}{c}\text { ABR } \\
(\%)\end{array}$ & $\begin{array}{l}\text { FR } \\
\text { (\%) }\end{array}$ & VIR \\
\hline \multicolumn{7}{|l|}{ PNEC } \\
\hline Abies religiosa (Kunth) Schltdl. \& Cham. & 454 & 55.23 & 259966.32 & 96.06 & 10.64 & 53.98 \\
\hline Juniperus monticola Martínez & 84 & 10.22 & 1314.53 & 0.49 & 10.64 & 7.11 \\
\hline Prunus serotina Ehrh. & 58 & 7.05 & 934.92 & 0.35 & 10.64 & 6.01 \\
\hline Lonicera mexicana (Kunth) Rehder & 50 & 6.08 & 291.29 & 0.11 & 8.51 & 4.9 \\
\hline Ribes ciliatum Humb. \& Bonpl. ex Roem. \& Schult. & 40 & 4.87 & 111.48 & 0.04 & 8.51 & 4.47 \\
\hline Quercus glabrescens Benth. & 26 & 3.16 & 6387.92 & 2.36 & 6.38 & 3.97 \\
\hline Roldana angulifolia (DC.) H. Rob. \& Brettell & 24 & 2.92 & 90.26 & 0.03 & 8.51 & 3.82 \\
\hline \multicolumn{7}{|l|}{ Ejido El Cerezo } \\
\hline Abies religiosa (Kunth) Schltdl. \& Cham. & 452 & 68.07 & 281749.12 & 96.08 & 18.52 & 60.25 \\
\hline Juniperus monticola Martínez & 64 & 9.64 & 1419.16 & 0.48 & 11.11 & 6.69 \\
\hline Alnus jorulensis Kunth & 4 & 0.6 & 11.98 & 0.004 & 3.7 & 5.79 \\
\hline Quercus rugosa Née & 10 & 1.51 & 672.4 & 0.23 & 7.41 & 3.9 \\
\hline Ribes ciliatum Humb. \& Bonpl. ex Roem. \& Schult. & 20 & 3.01 & 97.15 & 0.03 & 7.41 & 3.23 \\
\hline \multicolumn{7}{|l|}{ Ejido Pueblo Nuevo } \\
\hline Abies religiosa (Kunth) Schltdl. \& Cham. & 258 & 45.91 & 239040.09 & 91.2 & 15.15 & 50.75 \\
\hline Quercus glabrescens Benth. & 66 & 11.74 & 6969.73 & 2.66 & 9.09 & 7.83 \\
\hline Archibaccharis serratifolia (Kunth) S.F. Blake & 96 & 17.08 & 422.31 & 0.16 & 3.03 & 6.76 \\
\hline Prunus serotina Ehrh. & 30 & 5.34 & 3607.61 & 1.38 & 12.12 & 6.28 \\
\hline Lonicera mexicana (Kunth) Rehder & 28 & 4.98 & 135.28 & 0.05 & 9.09 & 4.71 \\
\hline Quercus rugosa Née & 24 & 4.27 & 1216.08 & 0.46 & 6.06 & 3.6 \\
\hline Pinus teocote Schltdl. \& Cham. & 4 & 0.71 & 6979.26 & 2.66 & 6.06 & 3.15 \\
\hline \multicolumn{7}{|l|}{ Ejido Xolostitla } \\
\hline Abies religiosa (Kunth) Schltdl. \& Cham. & 600 & 86.95 & 2024407.83 & 86.95 & 31.25 & 68.39 \\
\hline Pinus teocote Schltdl. \& Cham. & 16 & 2.32 & 21405.22 & 9.2 & 12.5 & 8 \\
\hline Quercus rugosa Née & 14 & 2.03 & 1776.2 & 0.76 & 18.75 & 7.18 \\
\hline Prunus serotina Ehrh. & 16 & 2.32 & 535.02 & 0.23 & 18.75 & 7.1 \\
\hline Quercus laurina Bonpl. & 28 & 4.06 & 3218.25 & 1.38 & 6.25 & 3.9 \\
\hline \multicolumn{7}{|l|}{ Ejido Tecocomulco } \\
\hline Abies religiosa (Kunth) Schltdl. \& Cham. & 950 & 60.2 & 245762.43 & 86.67 & 11.63 & 52.83 \\
\hline Quercus laurina Bonpl. & 236 & 14.95 & 14130.07 & 4.98 & 11.63 & 10.52 \\
\hline Ribes ciliatum Humb. \& Bonpl. ex Roem. \& Schult. & 110 & 6.97 & 411.9 & 0.15 & 11.63 & 6.25 \\
\hline Quercus crassifolia Bonpl. & 66 & 4.18 & 8268.87 & 2.92 & 11.63 & 6.24 \\
\hline Lonicera mexicana (Kunth) Rehder & 88 & 5.57 & 668.71 & 0.24 & 11.63 & 5.81 \\
\hline Pinus teocote Schltdl. \& Cham. & 10 & 0.63 & 11890.5 & 4.19 & 6.98 & 3.93 \\
\hline Arbutus xalapensis Kunth & 32 & 2.02 & 791.33 & 0.28 & 9.3 & 3.97 \\
\hline
\end{tabular}

Grupo 1. Formado por las localidades PNEC y El Cerezo, cercanas geográficamente entre sí; contiene la mayor riqueza de especies de árboles y arbustos (23). La especie dominante fue Abies religiosa y las especies codominantes en el dosel fueron Alnus jorullensis, Cupressus lusitanica Mill., Prunus serotina Ehrh. y Quercus spp. Las especies con VIR bajos aparecieron en condiciones particulares; por ejemplo, Salix paradoxa Kunth fue común en ambientes ri- 
parios, en cambio Rhamnus mucronata Schltdl. y $R$. serrata Schult. solo se encontraron en dos unidades de muestreo. Las especies arbustivas Juniperus monticola, Lonicera mexicana (Kunth) Rehder, Ribes ciliatum Humb. \& Bonpl. ex Roem. \& Schult. y Roldana angulifolia (DC.) H. Rob. \& Brettell fueron escasas debido a la alta cobertura del dosel (Fig. 5). El promedio de diámetro y altura en árboles fue de $21.01 \pm 23.06 \mathrm{~cm}$ y $14.41 \pm 13.11 \mathrm{~m}$, respectivamente. Sin embargo, algunos individuos de $A$. religiosa presentaron alturas de $51 \mathrm{~m}$ y diámetros de hasta $111 \mathrm{~cm}$. En este grupo se encontró el número más elevado de tocones y árboles caídos (principalmente en El Cerezo). Ambas localidades se encuentran en el límite altitudinal superior de distribución del bosque de oyamel en el estado de Hidalgo, por arriba de los $3000 \mathrm{~m}$.

Grupo 2. Incluye solo a Pueblo Nuevo donde se identificaron 18 especies de árboles y arbustos. Abies religiosa fue la especie dominante en el dosel, pero presentó el VIR más bajo comparado con el estimado en el resto de las localidades. Otras especies de árboles comunes en el dosel fueron Quercus glabrescens y Q. laurina. En el sotobosque predominó Archibaccharis serratifolia e individuos juveniles de A. religiosa (Fig. 6). El diámetro y altura promedio de las especies de árboles fue de $25.12 \pm 23.54 \mathrm{~cm}$ y $17.22 \pm 13.42$ $\mathrm{m}$, respectivamente. Algunos individuos de oyamel presentaron hasta $45 \mathrm{~m}$ de altura y $114 \mathrm{~cm}$ de diámetro. La cobertura del dosel del bosque fue abierta debido a la presencia frecuente de claros, con señales evidentes de disturbio a causa de actividades humanas (pastoreo, tala, presencia de caminos).

Grupo 3. Constituido por el bosque de Xolostitla, presentó la menor riqueza con solo siete especies. La especie arbórea dominante fue Abies religiosa y las especies codominantes pertenecen a los géneros Juniperus L., Pinus L., Prunus L. y Quercus (Fig. 7). El promedio de diámetro y altura de los árboles fue de $17.88 \pm 23.25 \mathrm{~cm}$ y $11.89 \pm 10.41 \mathrm{~m}$, respectivamente. Los árboles más robustos de $A$. religiosa alcanzaron alturas de hasta $48 \mathrm{~m}$ y $113 \mathrm{~cm}$ de diámetro. Existen claras evidencias de manejo dentro del bosque, debido a que los ejidatarios desarrollan labores de limpieza de hierbas y arbustos en sitios aledaños, pues existe un centro recreativo (ecoturismo) en el lugar.

Grupo 4. Formado por Tecocomulco, es la localidad más lejana geográficamente y con menor similitud florística y estructural, con respecto a las demás. Se identificaron 14 especies de árboles y arbustos; la especie dominan-

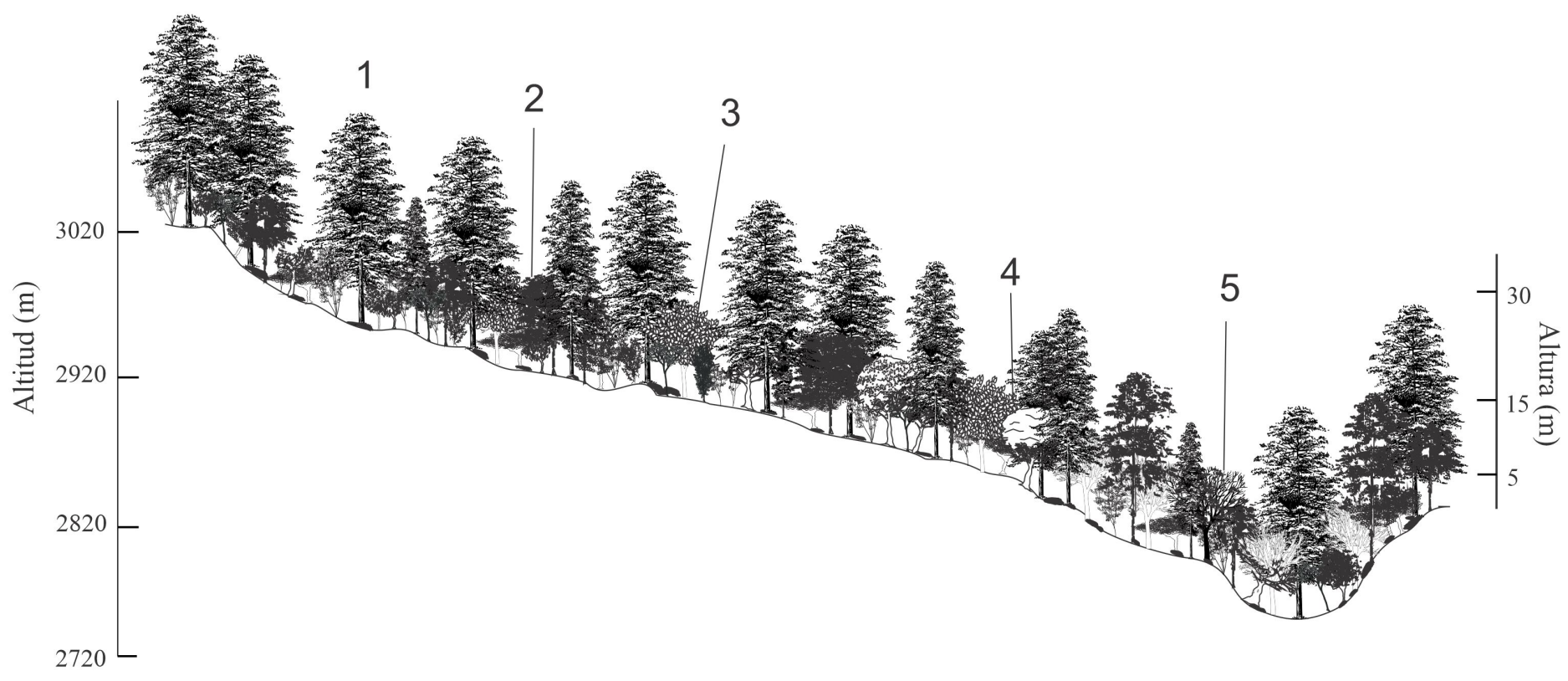

Figura 5: Diagrama de perfil semi-realista de las localidades PNEC y ejido El Cerezo (Grupo 1). 1. Abies religiosa (Kunth) Schltdl. \& Cham.; 2. Juniperus monticola Martínez; 3. Prunus serotina Ehrh.; 4. Quercus rugosa Née, 5. Alnus jorulensis Kunth. 


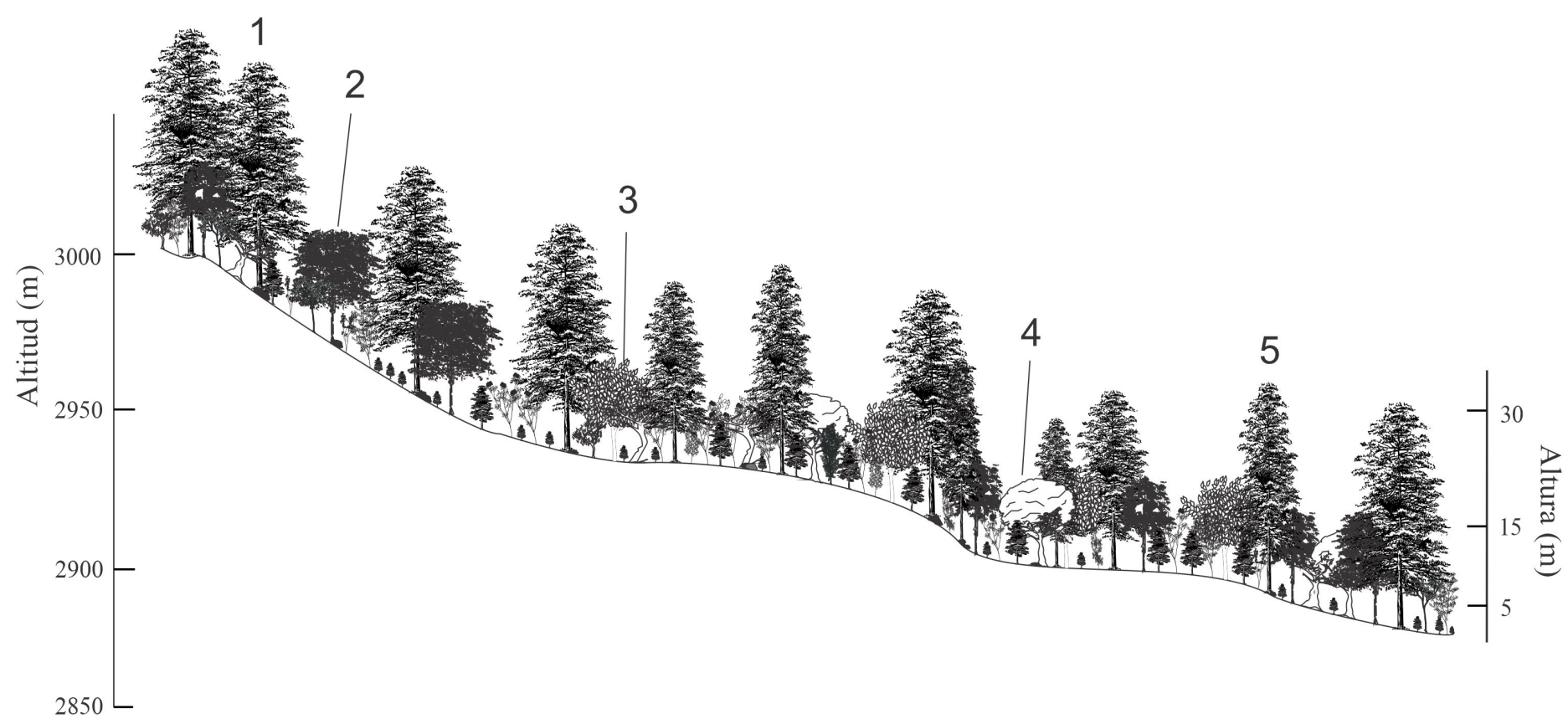

Figura 6: Perfil semi-realista del bosque de oyamel del ejido Pueblo Nuevo (Grupo 2). 1. Abies religiosa (Kunth) Schltdl. \& Cham.; 2. Juniperus monticola Martínez; 3. Prunus serotina Ehrh.; 4. Quercus glabrescens Benth.; 5. Pinus teocote Schltdl. \& Cham.

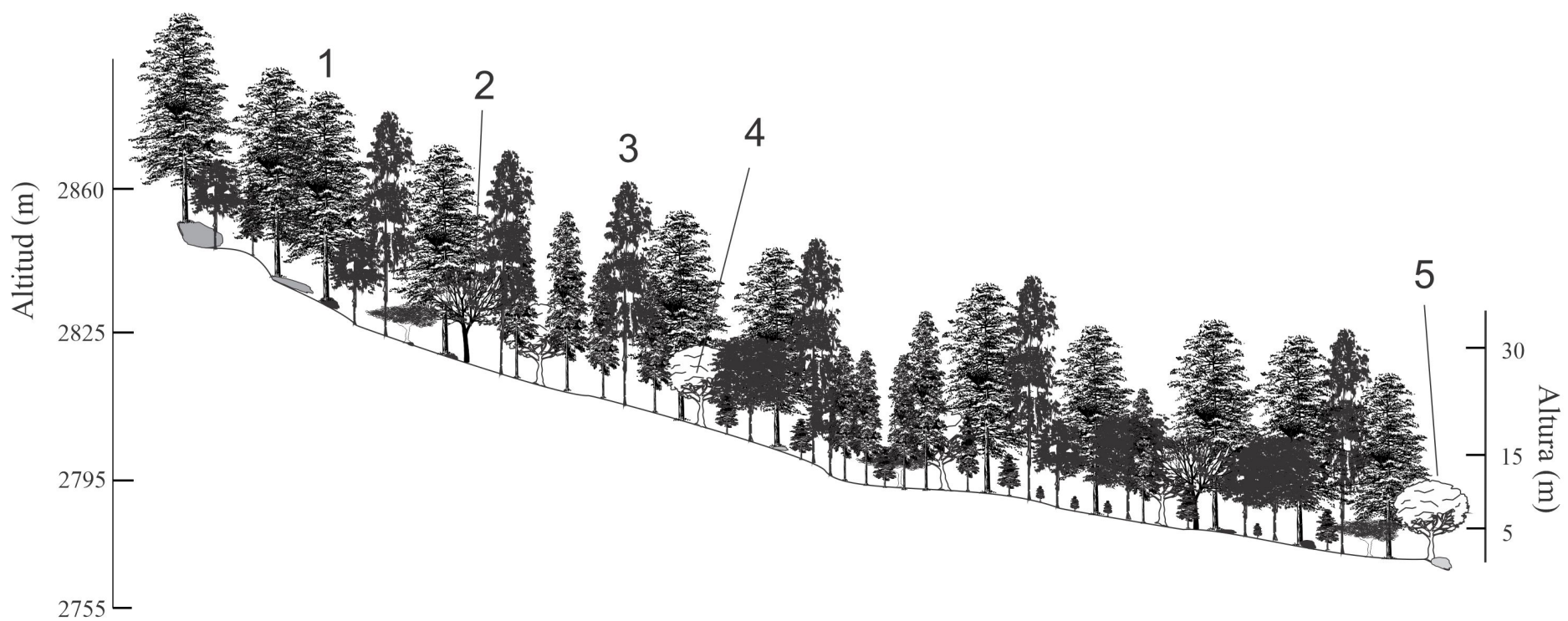

Figura 7: Perfil semi-realista del bosque del ejido Xolostitla (Grupo 3). 1. Abies religiosa (Kunth) Schltdl. \& Cham.; 2. Prunus serotina Ehrh.; 3. Pinus teocote Schltdl. \& Cham.; 4. Quercus rugosa Née; 5. Quercus laurina Bonpl.

te en el dosel fue $A$. religiosa y las codominantes fueron Pinus teocote y Quercus spp. y en el subdosel Juniperus monticola, Arbutus xalapensis Kunth, Buddleja cordata Kunth, Lonicera mexicana y Ribes ciliatum (Fig. 8). Los indicios de actividades humanas en esta localidad incluyen presencia de brechas corta fuegos y caminos hacia los po- blados cercanos. Aunque existe veda en el aprovechamiento de oyamel, se lleva a cabo la tala de algunas especies de Pinus. El promedio de diámetro y la altura de los árboles fue de $13.30 \pm 16.76 \mathrm{~cm}$ y $12.40 \pm 9.02 \mathrm{~m}$, respectivamente. Algunos individuos de oyamel poseían alturas de hasta $50 \mathrm{~m}$ y diámetros de hasta $110 \mathrm{~cm}$. 


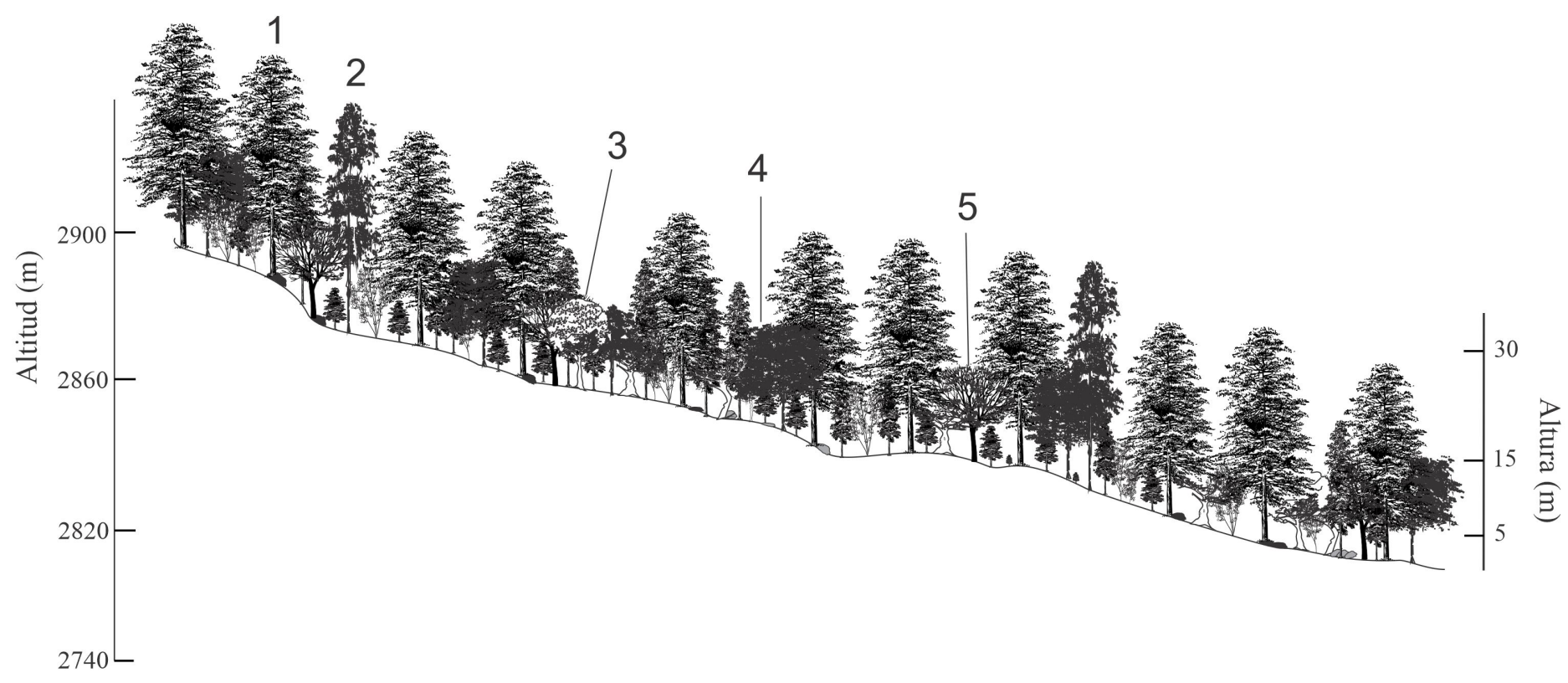

Figura 8: Perfil semi-realista del bosque de oyamel del ejido Tecocomulco (Grupo 4). 1. Abies religiosa (Kunth) Schltdl. \& Cham.; 2. Pinus teocote Schltdl. \& Cham.; 3. Quercus laurina Bonpl.; 4. Ribes ciliatum Humb. \& Bonpl. ex Roem. \& Schult.; 5. Arbutus xalapensis Kunth.

\section{Discusión}

\section{Estructura poblacional de Abies religiosa}

En la mayoría de las localidades la distribución de los individuos se concentró en las clases de altura y diámetro inferiores, por lo que prevaleció un patrón de "J" invertida representando un alto porcentaje de plantas juveniles. Por ello, las poblaciones se determinaron como dinámicas o en etapa de regeneración natural activa (Aguraiuja et al., 2008). El mismo patrón de desarrollo fue observado en otras poblaciones del género Abies (Sánchez-Velásquez et al., 1991; Cuevas-Guzmán et al., 2011; Pineda-López et al., 2013). Algunos autores plantean que la dominancia de individuos jóvenes es un indicio de una eficiente regeneración, establecimiento y por lo tanto reclutamiento, pero se trata de bosques distribuidos en pendientes pronunciadas y de difícil acceso para el hombre (Sánchez-Velásquez et al., 1991). En cambio, Pineda-López et al. (2013) señaIaron la posibilidad de que el alto número de plantas jóvenes se relacione con la extracción de especies forestales para diversos usos. Acorde con lo que mencionan estos autores, la ocurrencia de tres poblaciones dinámicas en la presente investigación probablemente se debe al manejo del bosque (leve a moderado), pues se trata de poblaciones situadas dentro de un ANP o bien donde existe veda para el oyamel, y en ambos casos permanecen en constante vigilancia.

El manejo no solo afecta la distribución de las plantas jóvenes o más robustas en tamaño, también tiende a cambiar la distribución de las plántulas $(<3.5 \mathrm{~cm})$. En este sentido, la mayor densidad de plántulas se observó en el ejido Pueblo Nuevo donde el manejo forestal es intensivo mientras que en el PNEC ocurre lo contrario. Un patrón similar fue descrito para los bosques de oyamel en Veracruz donde Lara-González et al. (2009) descubrieron una fuerte relación entre el número de plántulas y la edad del claro; es decir, la densidad de plántulas es menor en el sotobosque que en los espacios abiertos. Entonces, es posible que la alta densidad de plántulas en el sotobosque de la localidad de Pueblo Nuevo (población dinámica) esté relacionada con el aumento en la incidencia de luz, por la apertura continua del dosel debido al régimen de disturbio antrópico (apertura de claros). Al mismo tiempo, el aprovechamiento de diferentes especies arbóreas y arbustivas y la veda del oyamel, benefician la supervivencia de los árboles de Abies jóvenes y maduros, como se ha sugerido en otras especies de árboles de clima templado, como Fagus grandifolia subsp. mexicana (Martínez) A.E. Murray (Ortiz-Quijano et al., 2016). 
Aunado a los efectos antrópicos en los bosques, existe preocupación por las fases críticas del ciclo de vida de los árboles; principalmente en la germinación de las semillas y supervivencia de las plántulas. En el oyamel las semillas se caracterizan por su condición efímera, por lo que pierden su viabilidad el mismo año de su dispersión, lo cual impide la formación de un banco de semillas (Ángeles-Cervantes y López-Mata, 2009). Con relación a la fase de supervivencia de las plántulas de $A$. religiosa, los individuos pequeños son potencialmente susceptibles a plagas, enfermedades e incendios (Sánchez-Velásquez et al., 1991), pero se desconoce si sus semillas o plántulas son consumidas por herbívoros (Ángeles-Cervantes y López-Mata, 2009).

Con respecto a la correlación entre altura y diámetro, en especies arbóreas y en particular en Abies religiosa, se ha corroborado la existencia de una relación alométrica, por lo que el diámetro puede ser idóneo para estimar la altura de los árboles y viceversa (Li et al., 2015; Ortiz-Quijano et al., 2016). La relación alométrica de Abies religiosa en el presente estudio se obtuvo descartando aquellos individuos con valores de diámetro $<2.5 \mathrm{~cm}$ debido a que su patrón de crecimiento es más acelerado y diferente al presentado por los individuos de mayor edad (Vospernik et al., 2010; Ortiz-Quijano et al., 2016). En consecuencia, el resultado muestra un sesgo hacia clases de tamaño y diámetro mayores.

\section{Estructura del bosque de oyamel}

En el presente estudio solo se recolectaron e identificaron las especies de arbustos y árboles con mayor importancia estructural en el bosque de oyamel (A. religiosa). Por ello, la riqueza florística fue relativamente baja (30 especies) comparada con la estimada en el mismo tipo de vegetación para distintas entidades del país, considerando solo árboles y arbustos, como Coahuila (19 especies: Encina-Domínguez et al., 2008); Estado de México (33 especies: Sánchez-González et al., 2006 y 51 especies: Zepeda-Gómez et al., 2018); Jalisco (64 especies: Cisneros Lepe, 2005; Cuevas-Guzmán et al., 2011) y bosque de oyamel dominado por A. flinckii Rushforth en Jalisco (84 especies: Guerrero-Hernández et al., 2014). La diferencia en la riqueza de especies entre bosques ha sido relacionada con la ubicación geográfica, lo cual permite una conjugación especifica de las variables ambientales derivadas de la historia biogeográfica y geológica de la FVT (Rzedowski, 1978, 1998; Sánchez-González y López-Mata, 2003; Sánchez-González et al., 2006; EncinaDomínguez et al., 2008), con la presencia de otros tipos de vegetación aledaños con alta riqueza de especies, como el bosque mesófilo de montaña (Cisneros Lepe, 2005; CuevasGuzmán et al., 2011; Guerrero-Hernández et al., 2014), o bien con los diferentes criterios de delimitación y análisis de las comunidades consideradas como bosque de oyamel en cada investigación (Sánchez-González et al., 2005; Zepeda-Gómez et al., 2018).

A nivel local, el bosque de Xolostitla presentó la menor riqueza de especies. Esto puede estar relacionado con el disturbio antrópico derivado de las labores de limpieza del sotobosque (deshierbe) para fomentar el ecoturismo, que igualmente ha inducido la apertura de claros grandes. Aunado a ello, la importancia estructural de Abies más elevada que en las otras localidades tiene, tal vez, como causa principal la eliminación selectiva de especies de árboles y arbustos codominantes en áreas poco perturbadas, pues el uso del oyamel está vedado (Endara-Agramont y HerreraTapia, 2016). A diferencia del bosque de Xolostitla, en Tecocomulco, El Cerezo y el Parque Nacional El Chico, la riqueza de especies de arbustos y árboles fue más alta, en parte porque estas localidades comparten taxa con otras asociaciones vegetales contiguas, como el bosque de Pinus y de Quercus (Rzedowski, 1978; Sánchez González et al., 2005; Encina-Domínguez et al., 2008).

Las especies de arbustos y árboles identificadas en el presente estudio son representativas del bosque de oyamel de la FVT, ya que más de $80 \%$ de ellas han sido registradas en otros estudios de esta comunidad en la misma región biogeográfica (Rzedowski, 1978; Sánchez-González y López-Mata, 2003; Sánchez-González et al., 2005; 2006). A pesar de la similitud florística con otros bosques de Abies religiosa se observaron diferencias en los valores estructurales de las especies entre localidades de muestreo (presente estudio) y entre regiones (Sánchez-González y LópezMata, 2003; Encina-Domínguez et al., 2008; Bautista, 2013; González Cubas et al., 2017).

Entre las localidades analizadas, la importancia ecológica relativa de $A$. religiosa fluctúo entre 50.75 y $68.39 \%$. Los valores de VIR del oyamel parecen depender de la in- 
tensidad de manejo del bosque; ya que los valores más bajos se obtuvieron en las localidades con manejo moderado e intensivo (Pueblo Nuevo y Tecocomulco) y los valores más altos corresponden a localidades con manejo forestal leve y moderado (El Cerezo, Parque Nacional El Chico y Xolostitla). Por ello, el VIR del oyamel podría ser utilizado como indicador de la etapa de la sucesión ecológica y/o nivel de manejo del bosque (Cuadro 2). EI VIR de Abies religiosa, estimado en distintas regiones del país, es variable: en la Sierra Nevada, Estado de México, el valor fluctuó entre 17 y 30\% (Sánchez-González y López-Mata, 2003), en dos localidades de Tlaxcala el VIR fue de 37.9 y $55.3 \%$ (Bautista, 2013), en los bosques de Abies de Jalisco la variación entre sitios fue de 0.18 a $56.51 \%$, en el estado de Coahuila el VIR en Abies vejarii Martínez fue de $38.41 \%$ (Encina-Domínguez et al., 2008) y en Nuevo León, en distintas localidades con bosque de $A$. vejarii, fluctuó entre 16.9 y 29.4\% (González Cubas et al., 2017). Lo anterior sugiere, en primera instancia, que la estructura del bosque de oyamel cambia de forma drástica entre regiones, probablemente por la variación en las condiciones ambientales locales; por ejemplo, en altitud (temperatura y humedad), exposición, pendiente, grado de perturbación, condición de manejo, entre otros (Sánchez-González et al., 2005; Rodríguez-Laguna et al., 2015; Endara-Agramont y Herrera-Tapia, 2016). Sin embargo, desde el punto de vista metodológico, el tamaño y número de unidades de muestreo, además de las formas de vida de las especies consideradas en la estimación del VIR (árboles, arbustos, arboles, bejucos), fueron diferentes en cada investigación, por lo que no es adecuado comparar los valores estructurales del bosque de oyamel, entre las mismas.

Las cuatro variantes del bosque de oyamel reconocidas con el análisis de agrupamiento presentaron diferencias entre sí en los valores estructurales de las especies dominantes y codominantes, en la distancia geográfica y en el grado de manejo, que fue representado visualmente en los perfiles semi-realistas. Aunado a lo anterior, la riqueza y la codominancia cambian conforme se incrementa el gradiente de altitud: el bosque de oyamel del Parque Nacional El Chico y El Cerezo, destacan al poseer la mayor riqueza de árboles y arbustos codominantes y la presencia de árboles robustos de Abies religiosa en altura y diámetro. Esto coin- cide con lo observado en estudios previos, que sugieren que las poblaciones de esta especie de oyamel en México alcanzan su óptimo desarrollo en altitudes elevadas, entre 3200 y 3400 m (Sánchez-González et al., 2006; EncinaDomínguez et al., 2008). Es importante mencionar que el bosque de oyamel analizado se distribuye en el límite altitudinal inferior (entre 2320 y 3090 m), por lo que además de la altitud otros factores ambientales locales deben estar influyendo en los cambios de los valores de dominancia de A. religiosa (Sánchez-González et al., 2005; Rodríguez-Laguna et al., 2015; Zepeda-Gómez et al., 2018). De acuerdo con Vila et al. (2008) y Housset et al. (2016), cerca de los límites extremos de la distribución natural de las especies, el clima puede convertirse en un factor limitante para procesos fisiológicos importantes y tener un fuerte impacto sobre el crecimiento de las plantas.

El bosque de oyamel de Pueblo Nuevo puede utilizarse como ejemplo del papel de los factores locales sobre la estructura y composición de la vegetación, pues se caracteriza por el aprovechamiento forestal, el cambio de uso del suelo, la apertura de caminos y veredas fomentado por los ejidatarios. Lo anterior se ve reflejado en la presencia de especies indicadoras de disturbio reciente como Archibaccharis serratifolia, Baccharis conferta Kunth y Roldana spp. (Rzedowski, 1978; García-Sánchez et al., 2014; Rodríguez-Laguna et al., 2015), en la escasa densidad de individuos juveniles y adultos de Abies y en la alta densidad de renuevos de oyamel producto de la germinación de las semillas estimulada por la luz solar (Lara-González et al., 2009). Aunado a lo anterior, fue el bosque situado a menor altitud, con presencia de individuos adultos de oyamel menos robustos y con dosel codominado por especies representativas de tipos de vegetación aledaños como el bosque de Quercus y de Pinus (Rzedowski, 1978; Sánchez-González et al., 2005; Endara-Agramont y Herrera-Tapia, 2016).

\section{Conclusiones}

Esta investigación amplía el conocimiento actual sobre los cambios en la composición y estructura del bosque de Abies religiosa, los resultados indican un patrón de dominancia semejante entre las localidades analizadas. La variación detectada en las características estructurales a nivel poblacional y comunitario podría estar asociada con el manejo fo- 
restal en cada bosque y con los cambios en las condiciones ambientales locales si bien, futuros estudios son necesarios para confirmar esto. A nivel poblacional, la estructura del tamaño (altura) y diámetro de los individuos de A. religiosa permitió definir a las poblaciones como dinámicas (El Chico, Xolostitla, Tecocomulco), es decir, con predominio de individuos jóvenes y como normales (El Cerezo y Pueblo Nuevo), con preponderancia de individuos de tamaños intermedios. La relación alométrica entre el diámetro y la altura de los árboles, indica que una u otra característica se puede emplear para representar la estructura de tallas de las poblaciones de Abies. La estructura ecológica básica del bosque de oyamel, definida por pocas especies de árboles y arbustos, ayudó a reconocer cuatro grupos o variantes, que representaron distintas etapas de regeneración. El grupo 1 incluyó el bosque del Parque Nacional El Chico y El Cerezo, ambos se desarrollaban en condiciones fisiográficas similares y presentaron alta riqueza de especies; el grupo dos es el bosque del ejido Pueblo Nuevo, con manejo forestal y presentó señales de disturbio antrópico y baja riqueza de especies, el tercer grupo formado por el bosque de Xolostitla, con manejo forestal y destinado al ecoturismo, tuvo el menor número de especies. El cuarto grupo, formado por el bosque de Tecocomulco, con manejo forestal, presentó un número intermedio de especies. El bosque de oyamel del estado de Hidalgo no muestra aun indicios de cambios drásticos en su composición y estructura ecológica a pesar del manejo forestal.

\section{Contribución de autores}

ASG diseñó el estudio, AGH lo desarrolló e identificó las especies; AGH, JLR y ASG realizaron trabajo en campo y analizaron los resultados. JLR elaboró el mapa y JVD brindó apoyo y asesoría durante el desarrollo del trabajo. Todos los autores contribuyeron a la discusión, revisión y aprobación del manuscrito final.

\section{Financiamiento}

Esta investigación fue apoyada por el Consejo Nacional de Ciencia y Tecnología (CONACyT), proyecto Ciencia Básica CB2016/284484: "Efecto del cambio climático sobre poblaciones relictas de árboles; integrando dendrocronología y genética de poblaciones" y por la beca de Maestría 604934 a AGH.

\section{Agradecimientos}

Se agradece al programa de Maestría en Ciencias en Biodiversidad y Conservación de la Universidad Autónoma del Estado de Hidalgo, a Jesús Granados Lugo, director del Parque Nacional El Chico en el año 2017-2018, a los ejidatarios de El Cerezo y Pueblo Nuevo, a don Miguel Ortiz, don Reyes y don Julián del ejido Xolostitla y a doña Claudia, don José, don Tomás y don Guillermo Elizalde del ejido Tecocomulco. En la recolección de ejemplares de plantas, se contó con el permiso de colecta SEMARNAT, Oficio No. SGPA/ DGVS/003509/18.

\section{Literatura citada}

Aguraiuja, R., M. Moora y M. Zobel. 2004. Population stage structure of Hawaiian endemic fern taxa of Diellia (Aspleniaceae): Implications for monitoring and regional dynamics. Canadian Journal of Botany 82(10): 1438-1445. DOI: https://doi.org/10.1139/b04-105

Aguraiuja, R., M. Zobel, K. Zobel y M. Moora. 2008. Conservation of the endemic fern lineage Diellia (Aspleniaceae) on the Hawaiian Islands: can population structure indicate regional dynamics and endangering factors? Folia Geobotánica 43(3): 3-18. DOI: https://doi.org/10.1007/s12224-0089001-1

Alvarado, D., L. I. Bauer y A. Galindo. 1993. Decline of sacred fir (Abies religiosa) in a forest park south of Mexico City. Environmental Pollution 80(2): 115-121. DOI: https://doi. org/10.1016/0269-7491(93)90136-C

Ángeles-Cervantes, E. y L. López-Mata. 2009. Supervivencia de una cohorte de plántulas de Abies religiosa bajo diferentes condiciones de post-incendio. Boletín de la Sociedad Botánica de México 84: 25-33. DOI: https://doi. org/10.17129/botsci.2289

Aquino-Ramírez, M., A. Velázquez-Martínez, J. Villanueva-Díaz, H. L. Hervert-Zamora, A. Gómez-Guerrero, V. J. ReyesHernández y G. Ramírez-Valverde. 2019. Respuesta climática de Abies guatemalensis Rehder en Ixtlán de Juárez, Oaxaca, México. Madera y Bosques 25(2): e2521773. DOI: https:// doi.org/10.21829/myb.2019.2521773

Barrios-Rodríguez, M. A. y J. M. Medina-Cota. 1996. Estudio florístico de la Sierra de Pachuca, Estado de Hidalgo, México. Instituto Politécnico Nacional, Escuela Nacional de Ciencias Biológicas. México, D.F., México. 140 pp. 
Bautista, S. C. 2013. Estructura del bosque y arquitectura de brinzales de Abies religiosa en Tlaxco, Tlaxcala. Tesis de maestría. Postgrado Forestal, Instituto de Enseñanza e Investigación en Ciencias Agrícolas, Campus Montecillo, Colegio de Postgraduados. Montecillo, México. 115 pp.

Bianchi, L., A. Bottacci, G. Calamini, A. Maltoni, B. Mariotti, G. Quilghini, F. Salbitano, A. Tani, A. Zoccola y M. Paci. 2011. Structure and dynamics of a beech forest in a fully protected area in the northern Apennines (Sasso Fratino, Italy). iForest-Biogeosciences and Forestry 4(3): 136-144. DOI: https://doi.org/10.3832/ifor0564-004

Bravo Bolaños, O., A. Sánchez-González, J. A. Nova Vázquez y N. P. Pavón Hernández. 2016. Composición y estructura arbórea y arbustiva de la vegetación de la zona costera de Bahía de Banderas, Nayarit, México. Botanical Sciences 94(3): 603623. DOI: https://doi.org/10.17129/botsci.461

Calderón de Rzedowski, G. y J. Rzedowski. 2001. Flora fanerogámica del Valle de México. 2a Ed. Instituto de Ecología y Comisión Nacional para el Conocimiento y Uso de la Biodiversidad. Pátzcuaro, México. 1406 pp.

Carbajal-Navarro, A., E. Navarro-Miranda, A. Blanco-García, A. L. Cruzado-Vargas, E. Gómez-Pineda, C. ZamoraSánchez, F. Pineda-García, G. O’Neill, M. Gómez-Romero, R. Lindig-Cisneros, K. H. Johnsen, P. Lobit, L. LopezToledo, Y. Herrerías-Diego y C. Sáenz-Romero. 2019. Ecological Restoration of Abies religiosa Forests Using Nurse Plants and Assisted Migration in the Monarch Butterfly Biosphere Reserve, Mexico. Frontiers in Ecology and Evolution 7: 1-16. DOI: https://doi.org/10.3389/ fevo.2019.00421

Carlón-Allende, T., M. E. Mendoza, D. R. Pérez-Salicrup, J. Villanueva-Díaz y A. Lara. 2016. Climatic responses of Pinus pseudostrobus and Abies religiosa in the Monarch Butterfly Biosphere Reserve, Central Mexico. Dendrochronologia 38: 103-116. DOI: https://doi.org/10.1016/j. dendro.2016.04.002

Challenger, A. 1998. Utilización y Conservación de los Ecosistemas Terrestres de México, Pasado, presente y Futuro. Comisión Nacional para el Conocimiento y Uso de la BiodiversidadInstituto de Biología, Universidad Nacional Autónoma de México. México, D.F., México. 847 pp.

Cisneros Lepe, A. 2005. Estructura, composición florística y diversidad de los bosques de Abies en el estado de Jalisco,
México. Tesis de licenciatura. Universidad de Guadalajara. Autlán de Navarro, Jalisco, México. 101 pp.

CONANP. 2006. Programa de Conservación y Manejo. Parque Nacional El Chico, Secretaría de Medio Ambiente y Recursos Naturales. Cd. Mx., México. 182 pp.

Cuevas-Guzmán, R., E. A. Cisneros-Lepe, E. J. Jardel-Peláez, E. Sánchez-Rodríguez, L. Guzmán-Hernández, N. M. NúñezLópez y C. Rodríguez-Guerrero. 2011. Análisis estructural y de diversidad en los bosques de Abies de Jalisco, México. Revista Mexicana de Biodiversidad 82(4): 1219-1233. DOI: http://dx.doi.org/10.22201/ib.20078706e.2011.4.741

Curtis, J. T. y R. P. Mclntosh. 1951. An Upland Forest Continuum in the Prairie-Forest Border Region of Wisconsin. Ecology 32(3): 476-496. DOI: https://doi.org/10.2307/1931725

Daniel, W. W. 1995. Bioestadística, bases para el análisis de las ciencias de la salud. Editorial Limusa Wiley. México, D.F., México. 755 pp.

Encina-Domínguez, J. A., F. J. Encina-Domínguez, E. Mata-Rocha y J. Valdes-Reyna. 2008. Aspectos estructurales, composición florística y caracterización ecológica del bosque de oyamel de la Sierra de Zapalinamé, Coahuila, México. Boletín de la Sociedad Biológica Mexicana 83: 13-24.

Endara-Agramont, A. R. y F. Herrera-Tapia. 2016. Deterioro y conservación de los bosques del Nevado de Toluca y el rol de los actores locales. CIENCIA ergo-sum 23(3): 247-254.

Farjon, A. 2010. A handbook of the world's conifers. BRILL. Leiden, The Netherlands. $1111 \mathrm{pp}$.

Ferrusquía-Villafranca, I. 1998. Geología de México: una sinopsis. In: Ramamoorthy, T. P., R. Bye, A. Lot y J. Fa. (Comp.). Diversidad Biológica de México: Orígenes y Distribución. Instituto de Biología, Universidad Nacional Autónoma de México, México, D.F., México. Pp. 1-107.

Flores-Mata, G., L. J. Jiménez, S. X. Madrigal, R. F. Moncayo y T. F. Takaki. 1971. Memoria del mapa de tipos de vegetación de la República Mexicana. Secretaría de Recursos Hidráulicos. México, D.F., México. 50 pp.

Flores Nieves, P. 2015. Migración de Abies religiosa en gradientes altitudinales en el Cerro Tláloc. Tesis de doctorado. Colegio de Postgraduados. Montecillo, Texcoco, México. 63 pp.

Galindo-Leal, C., S. Franco, M. Flores, C. Lazcano, S. Franco y R. Cortés. 1988. Plan de Manejo Parque Nacional El Chico. Secretaría de Desarrollo Urbano y Ecología. Pachuca de Soto, México. 55 pp. 
García, E. 2004. Modificaciones al sistema de clasificación climática de Köppen. Instituto de Geografía. Universidad Nacional Autónoma de México. México, D.F., México. 91 pp.

García, M. H. 2002. Caracterización de hábitats que ameritan protección en el estado de Hidalgo. Tesis en Ingeniería Forestal con Orientación en Silvicultura. Universidad Autónoma Chapingo. Chapingo, México. 256 pp.

García-Sánchez, C. A., A. Sánchez-González y J. L. Villaseñor. 2014. La familia Asteraceae en el Parque Nacional Los Mármoles, Hidalgo, México. Acta Botanica Mexicana 106: 97-116. DOI: https://doi.org/10.21829/abm106.2014.219

Gentry, A. H. 1982. Patterns of Neotropical plant species diversity. In: Hecht, M. K., B. Wallace, G. T. Prance (eds.). Evolutionary Biology. Springer. Boston, USA. Pp. 1-84. DOI: https://doi. org/10.1007/978-1-4615-6968-8_1

González Cubas, R., E. J. Treviño Garza, M. A. González Tagle, A. Duque Montoya y A. Gómez Cárdenas. 2017. Diversidad y estructura arbórea en un bosque de Abies vejarii Martínez en el sur del estado de Nuevo León. Revista Mexicana de Ciencias Forestales 9(45): 36-65. DOI: https://doi. org/10.29298/rmcf.v9i45.142

González Hernández, A., R. Pérez Miranda, F. Moreno Sánchez, G. Ramírez Ojeda, S. Rosales Mata, A. Cano Pineda, V. Guerra de la Cruz y M. C. Torres Esquivel. 2015. Variabilidad de la temperatura local en bosques de coníferas por efectos de la deforestación. Revista Mexicana de Ciencias Forestales 6(31): 22-39. DOI: https://doi.org/10.29298/rmcf.v6i31.193

Guerrero-Hernández, R., J. G. González-Gallegos y A. CastroCastro. 2014. Análisis florístico de un bosque de Abies y el bosque mesófilo de montaña adyacente en Juanacatlán, Mascota, Jalisco, México. Botanical Sciences 92(4): 541562. DOI: https://doi.org/10.17129/botsci.119

Hammer, Ø., D. A. T. Harper y P. D. Ryan. 2001. PAST: Paleontological Statistics Software Package for Education and Data Analysis. Palaeontologia Electronica 4: 1-9.

Hegland, S. J., M. Van Leeuwen y J. G. B. Oostermeijer. 2001. Population structure of Salvia pratensis in relation to vegetation and management of Dutch dry floodplain grasslands. Journal of Applied Ecology 38(6): 1277-1289.

Hernández, M. E. 1985. Distribución y utilidad de los Abies de México. Boletín del Instituto de Geografía 15: 75-118.

Hernández, R. M. del R. 1995. Estudio florístico-fanerogámico del Parque Nacional El Chico, estado de Hidalgo. Tesis de licenciatura. Escuela Nacional de estudios Profesionales Iztacala, Universidad Nacional Autónoma de México. Tlalnepantla, México. 73 pp.

Housset, J. M., C. Carcaillet, M. P. Girardin, H. Xu, F. Tremblay y Y. Bergeron. 2016. In situ comparison of tree-ring responses to climate and population genetics: the need to control for local climate and site variables. Frontiers in Ecology and Evolution 4: 123. DOI: https://doi.org/10.3389/ fevo.2016.00123

INEGI. 2017. Instituto Nacional de Estadística, Geografía e Informática. Conjunto de Datos Vectoriales de Uso de Suelo y Vegetación. Escala 1:250 000. Serie VI. https://www.inegi. org.mx (consultado marzo de 2019).

IPNI. 2020. The International Plant Name Index. http://www.ipni. org (consultado febrero de 2020).

IUCN. 2020. The International Union for Conservation of Nature. Red List of Threatened Species, version 2020.1. http:// www.iucnredlist.org/ (consultado enero de 2020).

Kasischke, E. S. y B. J. Stocks. 2000. Fire, Climate Change, and Carbon Cycling in the Boreal Forest. Springer-Verlag. New York, USA. 464 pp.

Lamprecht, H. 1986. Waldbau in den Tropen. Verlag Paul Parey. Hamburg und Berlin, Alemania. 318 pp.

Lara-González, R., L. R. Sánchez-Velásquez y J. Corral Aguirre. 2009. Regeneration of Abies religiosa in canopy gaps versus understory, Cofre de Perote National Park, Mexico. Agrociencia 43(7): 739-747.

Li Y-q, X-w Deng, Z-h Huang, W-h Xiang, W-d Yan, P-f Lei, X-I Zhou y C-h Peng. 2015. Development and evaluation of models for the relationship between tree height and diameter at breast height for Chinese-fir plantations in subtropical China. PLoS ONE 10(4): e0125118. DOI: https://doi.org/10.1371/ journal.pone.0125118

Madrigal, S. X. 1967. Contribución al conocimiento de la ecología de los bosques de oyamel (Abies religiosa) (H. B. K.) Schl. et Cham.) en el Valle de México. Boletín Técnico 18. Instituto Nacional de Investigaciones Forestales. México, D.F., México. $94 \mathrm{pp}$.

Matteucci, S. D. y A. Colma. 1982. Metodología para el estudio de la vegetación. Secretaria General de la Organización de los Estados Americanos, 83 Programa Regional de Desarrollo Científico y Tecnológico. Washington, D.C., EUA. 168 pp. 
McCune, B. y J. B. Grace. 2002. Analysis of Ecological Communities. MjM Software Design. Gleneden Beach, USA.

Mueller-Dombois, D. y H. Ellenberg. 1974. Aims and Methods of Vegetation Ecology. John Wiley \& Sons. New York, USA. 547 pp.

Ortiz-Quijano, A. B., A. Sánchez-González, L. López-Mata y J. Villanueva-Díaz. 2016. Population structure of Fagus grandifolia subsp. mexicana in the cloud forest of Hidalgo State, Mexico. Botanical Sciences 94(3): 483-497. DOI: https://doi.org/10.17129/botsci.515

Pavón, N. P. y M. Meza. 2009. Cambio climático en el estado de Hidalgo: clasificación y tendencias climáticas. Universidad Autónoma del Estado de Hidalgo. Pachuca de Soto, México. 44 pp.

Pérez Miranda, R., F. Moreno Sánchez, A. González Hernández y V. J. Arriola Padilla. 2014. Distribución de Abies religiosa (Kunth) Schltdl. \& Cham. y Pinus montezumae Lamb. Ante el cambio climático. Revista Mexicana de Ciencias Forestales 5(25): 18-33. DOI: https://doi.org/10.29298/rmcf.v5i25.301

Pérez-Paredes, M. G., A. Sánchez-González y J. D. TejeroDíez. 2014. Estructura poblacional y características del hábitat de dos especies de Cyatheaceae del estado de Hidalgo. Botanical Sciences 92: 259-271. DOI: https://doi. org/10.17129/botsci.48

Pineda-López, M. del R., R. Ortega-Solís, L. R. Sánchez-Velásquez, G. Ortiz-Ceballos y G. Vázquez-Domínguez. 2013. Estructura poblacional de Abies religiosa (Kunth). Schltdl. \& Cham., en el ejido el Conejo del Parque Nacional Cofre de Perote, Veracruz, México. Revista Chapingo Serie Ciencias Forestales y del Ambiente 19(2): 375-385. DOI: http:// dx.doi.org/10.5154/r.rchscfa.2012.11.058

Razo-Zárate, R., A. J. Gordillo-Martínez, R. Rodríguez-Laguna, C. C. Maycotte-Morales y O. A. Acevedo-Sandoval. 2013. Escenarios de carbono para el bosque de oyamel del Parque Nacional El Chico, Hidalgo, México. Revista Latinoamericana de Recursos Naturales 9(1): 17-21.

Rodríguez-Laguna, R., R. Razo-Zárate, J. Fonseca-González, J. Capulín-Grande y R. Goche-Telles. 2015. Regeneración natural post-incendio de Abies religiosa (Kunth) Schltdl. et Cham., en el Parque Nacional “El Chico" Hidalgo. Revista Iberoamericana de Ciencias 2(2): 11-22.

Rozas, V. 2002. Estructura y patrones de regeneración del roble y el haya en un bosque maduro del litoral occidental de
Cantábrica. Investigación Agraria: Sistemas y Recursos Forestales 11: 109-138.

Rzedowski, J. 1978. Vegetación de México. Limusa, México, D.F. $432 \mathrm{pp}$.

Rzedowski, J. 1998. Diversidad y orígenes de la flora fanerogámica de México. In: Ramamoorthy, T. P., R. Bye, A. Lot y J. Fa (eds.). Diversidad biológica de México: orígenes y distribución, Instituto de Biología, Universidad Nacional Autónoma de México. México, D.F., México. Pp. 129-145.

Rzedowski, J. y G. Calderón de Rzedowski. 1979. Flora fanerogámica del Valle de México, Vol. I. Compañía Editorial Continental. México, D.F., México. 403 pp.

Sáenz-Romero, C., G. E. Rehfeldt, P. Duval y R. A. Lindig-Cisneros. 2012. Abies religiosa habitat prediction in climatic change scenarios and implications for monarch butterfly conservation in Mexico. Forest Ecology Management 275: 98-106. DOI: https://doi.org/10.1016/j.foreco.2012.03.004 Sánchez-González, A. y L. López-Mata. 2003. Clasificación y ordenación de la vegetación del norte de la Sierra de Nevada, a lo largo de un gradiente altitudinal. Anales del Instituto de Biología, Serie Botánica 74(1): 43-71.

Sánchez-González, A. y L. López-Mata. 2005. Plant species richness and diversity along an altitudinal gradient in the Sierra Nevada, Mexico. Diversity and Distributions 11(6): 567-575. DOI: https://doi.org/10.1111/j.1366-9516.2005.00186.x

Sánchez-González, A., L. López-Mata y D. Granados-Sánchez. 2005. Semejanza florística entre los bosques de Abies religiosa (H.B.K.) Cham. \& Schltdl. de la Faja Volcánica Transmexicana. Boletín del Instituto de Geografía 56: 62-76. DOI: http://dx.doi.org/10.14350/rig.30097

Sánchez-González, A., L. López-Mata y H. Vibrans. 2006. Composición y patrones de distribución geográfica de la flora del bosque de oyamel del Cerro Tláloc, México. Boletín de la Sociedad Botánica de México 69: 67-78. DOI: https:// doi.org/10.17129/botsci.1734

Sánchez-Rodríguez, E. V., L. López-Mata, E. García-Moya y R. Cuevas-Guzmán. 2003. Estructura, composición florística y diversidad de especies leñosas de un bosque mesófilo de montaña en la Sierra de Manantlán, Jalisco. Boletín de la Sociedad Botánica de México 73: 17-34. DOI: https://doi. org/10.17129/botsci.1676

Sánchez-Velásquez, L. R., Ma. del R. Pineda-López y A. HernándezMartínez. 1991. Distribución y estructura de la población de 
Abies religiosa (H.B.K.) Schl. et Cham., en el Cofre de Perote, Estado de Veracruz, México. Acta Botanica Mexicana 16: 45-55. DOI: https://doi.org/10.21829/abm16.1991.625

Santibañez-Andrade, G., S. Castillo-Argüero y Y. MartínezOrea. 2015. Evaluación del estado de conservación de la vegetación de los bosques de una cuenca heterogénea del Valle de México. BOSQUE 36(2): 299-313. DOI: https://doi. org/10.4067/S0717-92002015000200015

Schmidt, T., P. Arens, M. J. M. Smulders, R. Billeter, J. Liira, I. Augenstein y W. Durka. 2009. Effects of landscape structure on genetic diversity of Geum urbanum L. populations in agricultural landscapes. Flora 204(7): 549-559. DOI: https:// doi.org/10.1016/j.flora.2008.07.005

SEMARNAT. 2010. Norma Oficial Mexicana NOM-059SEMARNAT-2010. Protección ambiental-Especies nativas de México de flora y fauna silvestres-Categorías de riesgo y especificaciones para su inclusión, exclusión o cambio-Lista de especies en riesgo. Secretaría del Medio Ambiente y Recursos Naturales. Diario Oficial de la Federación. Cd. Mx., México. http://dof.gob.mx/nota_detalle.php?codigo=5173 091\&fecha=30/12/2010 (consultado enero, 2020).

Statsoft. 2015. STATISTICA. Statsoft Dell Inc. Version 13.0.

Toledo, V. M., J. Carabias, C. Toledo y C. González-Pacheco. 1993. La Producción Rural en México: Alternativas Ecológicas. Fundación Universo Veintiuno y Prensa de Ciencias. México, D.F., México. 402 pp.

Trejo, I. y R. Dirzo. 2002. Floristic diversity of Mexican seasonally dry tropical forests. Biodiversity and Conservation 11: 20632048.
TROPICOS. 2020. Tropicos.org. Missouri Botanical Garden. http:// www.tropicos.org (consultado enero, 2020).

Urban, J., K. Rebrošová, L. Dobrovolný y J. Schneider. 2010. Allometry of four European beech stands growing at the contrasting localities in small-scale area. Folia Oecologica 37: 103-111.

Valencia-A., S., G. Flores-Franco, J. Jiménez-Ramírez y M. MoraJarvio. 2017. Distribution and diversity of Fagaceae in Hidalgo, Mexico. Botanical Sciences 95(4): 660-721. DOI: https://doi.org/10.17129/botsci.1020

Vila, B., M. Vennetier, C. Ripert, O. Chandioux y E. Liang. 2008. Has global change induced divergent trends in radial growth of Pinus sylvestris and Pinus halepensis at their bioclimatic limit? The example of the Sainte-Baume forest (south-east France). Annals of Forest Science 65:709. DOI: https://doi. org/10.1051/forest:2008048

Vospernik, S., R. A. Monserud y H. Sterba. 2010. Do individual tree growth models correctly represent height: diameter ratios of Norway spruce and Scots pine? Forest Ecology and Management 260(10): 1735-1753. DOI: https://doi. org/10.1016/j.foreco.2010.07.055

Zepeda-Gómez, C., C. Burrola-Aguilar y M. E. Estrada-Zúñiga. 2018. Riqueza y afinidades geográficas de la flora de un bosque de Abies religiosa de la Faja Volcánica Transmexicana. Caldasia 40(1): 54-70. DOI: http://dx.doi.org/10.15446/caldasia. v40n1.68658 
Apéndice. Listado de especies con su valor de importancia relativa (VIR), por localidad de muestreo en el bosque de Abies religiosa (Kunth) Schltdl. \& Cham. de Hidalgo, México. Forma biológica (FB); árbol (A); arbusto (Ar). Categoría de riesgo (NOM-059-2010, SEMARNAT, 2010): Protección especial (Pr); en peligro de extinción (P).

\begin{tabular}{|c|c|c|c|c|c|c|c|}
\hline Especie/Localidad & Familia & FB & PNEC & El Cerezo & $\begin{array}{l}\text { Pueblo } \\
\text { Nuevo }\end{array}$ & Xolostitla & Tecocomulco \\
\hline Abies religiosa (Kunth) Schltdl. \& Cham & Pinaceae & $A$ & 53.98 & 60.25 & 50.75 & 68.39 & 52.83 \\
\hline Alnus jorullensis Kunth & Betulaceae & $A$ & - & 5.79 & - & - & - \\
\hline Arbutus xalapensis Kunth & Ericaceae & $A$ & 2.8 & - & 1.14 & - & 3.87 \\
\hline Archibaccharis serratifolia (Kunth) S.F. Blake & Asteraceae & $\operatorname{Ar}$ & - & - & 6.76 & - & - \\
\hline Baccharis conferta Kunth & Asteraceae & $\operatorname{Ar}$ & 1.75 & 1.31 & 1.73 & - & - \\
\hline Berberis moranensis Schult. \& Schult. f. & Berberidaceae & $\operatorname{Ar}$ & - & - & 1.13 & - & - \\
\hline Buddleia cordata Kunth & Scrophulariaceae & $\mathrm{Ar}$ & - & - & 1.13 & - & 2.9 \\
\hline${ }^{\mathrm{Pr} C o m a r o s t a p h y l i s ~ d i s c o l o r ~(H o o k .) ~ D i g g s ~}$ & Ericaceae & $\operatorname{Ar}$ & 1.29 & 1.37 & - & - & - \\
\hline Crataegus mexicana DC. & Rosaceae & A & 0.87 & - & 2.9 & - & - \\
\hline${ }^{\mathrm{Pr} C u p r e s s u s ~ I u s i t a n i c a ~ M i l l . ~}$ & Cupressaceae & $A$ & - & 2.61 & - & - & - \\
\hline Fuchsia microphylla Kunth & Onagraceae & $\operatorname{Ar}$ & - & - & 1.26 & - & - \\
\hline Garrya laurifolia Hartw. ex Benth. & Garryaceae & $A$ & 2.72 & 1.38 & - & - & - \\
\hline Ilex tolucana Hemsl. & Aquifoliaceae & $\mathrm{Ar}$ & - & 1.21 & 1.13 & - & - \\
\hline PrJuniperus monticola Martínez & Cupressaceae & $\mathrm{Ar}$ & 7.11 & 6.69 & - & 2.91 & - \\
\hline${ }^{\mathrm{P}}$ Litsea glaucescens Kunth & Lauraceae & $\operatorname{Ar}$ & 2.54 & - & 1.13 & - & - \\
\hline Lonicera mexicana (Kunth) Rehder & Caprifoliaceae & $\operatorname{Ar}$ & 4.9 & - & 4.71 & - & 5.81 \\
\hline Pinus teocote Schltdl. \& Cham. & Pinaceae & A & - & - & 3.15 & 8 & 3.93 \\
\hline Prunus serotina Ehrh. & Rosaceae & A & 6.01 & 2.66 & 6.28 & 7.1 & 1.71 \\
\hline Quercus crassifolia Bonpl. & Fagaceae & A & - & 1.21 & 2.37 & 2.52 & 6.24 \\
\hline Quercus crassipes Bonpl. & Fagaceae & $A$ & - & - & - & - & 0.87 \\
\hline Quercus glabrescens Benth. & Fagaceae & A & 3.97 & 1.61 & 7.83 & - & - \\
\hline Quercus laeta Liebm. & Fagaceae & A & 1.23 & - & - & - & 1.69 \\
\hline Quercus laurina Bonpl. & Fagaceae & A & 1.75 & 2.37 & 1.29 & 3.9 & 10.52 \\
\hline Quercus rugosa Née & Fagaceae & A & - & 3.9 & 3.6 & 7.18 & 0.82 \\
\hline Rhamnus mucronata Schltdl. & Rhamnaceae & $\operatorname{Ar}$ & - & 1.21 & - & - & - \\
\hline Rhamnus serrata Schult. var. serrata & Rhamnaceae & $\operatorname{Ar}$ & 0.79 & - & - & - & - \\
\hline Ribes ciliatum Humb. \& Bonpl. ex Roem. \& Schult. & Grossulariaceae & $\operatorname{Ar}$ & 4.47 & 3.23 & - & - & 6.25 \\
\hline Roldana angulifolia (DC.) H. Rob. \& Brettell & Asteraceae & $\mathrm{Ar}$ & 3.82 & 1.92 & 1.73 & - & - \\
\hline Salix paradoxa Kunth & Salicaceae & A & - & 1.31 & - & - & 1.73 \\
\hline Symphoricarpos microphyllus Kunth & Caprifoliaceae & $\operatorname{Ar}$ & - & - & - & - & 0.82 \\
\hline
\end{tabular}

